\title{
FLUID-STRUCTURE INTERACTION BASED ON HPC MULTICODE COUPLING*
}

\author{
J. C. CAJAS $^{\dagger}$, G. HOUZEAUX ${ }^{\dagger}$, M. VÁZQUEZ ${ }^{\dagger}$, M. GARCÍA ${ }^{\dagger}$, E. CASONI $^{\dagger}$, \\ H. CALMET ${ }^{\dagger}$, A. ARTIGUES ${ }^{\dagger}$, R. BORRELL ${ }^{\dagger}$, O. LEHMKUHL $^{\dagger}$, D. PASTRANA ${ }^{\dagger}$, \\ D. J. YÁÑEZ§, R. PONS $₫$, AND J. MARTORELLף
}

\begin{abstract}
The fluid-structure interaction (FSI) problem has received great attention in the last few years, mainly because it is present in many physical systems, industrial applications, and almost every biological system. In the parallel computational field, outstanding advances have been achieved for the individual components of the problem, allowing, for instance, simulations around complex geometries at very high Reynolds numbers or simulations of the contraction of a beating heart. However, it is not an easy task to combine the advances of both fields, given that they have followed development paths in a rather independent way, and also because physical and numerical instabilities arise when dealing with two highly nonlinear partial differential equations. Nonetheless, in the last few years great advances in the coupled FSI field have been achieved, recognizing the most challenging problems to tackle and enabling a new generation of numerical simulations in aerodynamics, biological systems, and complex industrial devices. Keeping in mind that efficient parallel codes for the individual components already exist, this paper presents a framework to build a massively parallel FSI solver in a multicode coupling partitioned approach, with strong focus in the parallel implementation aspects and the parallel performance of the resulting application. The problem is casted in an algebraic form, and the main points of interest are the parallel environment needed to be able to transfer data among the codes, the location of the exchange surface, and the exchange of information among the parallel applications. The proposed framework has been implemented in the HPC multiphysics code Alya, and the multicode coupling is carried out running separated instances of this code. Two coupling algorithms with different acceleration schemes are revised, and three representative cases of different areas of interest showing the reach of the proposed framework are solved. Good agreement with literature and experiments is obtained. In addition to the numerical validation of the FSI solver, an assessment of the parallel performance of the proposed multicode strategy is done. In particular, a special distribution of the fluid code and solid code MPI processes on the supercomputer nodes based on computing cores overloading is investigated. Finally, a strong scalability test, running up to a 30 million elements case using 1280 MPI processes, is done.
\end{abstract}

Key words. fluid-structure interaction, HPC, multicode coupling

AMS subject classifications. 74F $10,68 \mathrm{U} 20,6804$

DOI. $10.1137 / 17 \mathrm{M} 1138868$

1. Introduction. Fluid-structure interaction (FSI) governs so many physical problems, industrial devices, and biological systems that its relevance is out of the question. FSI combines the challenges of computational fluid dynamics (CFD) with those of computational solid dynamics (CSD). Two very complete overviews of the

*Submitted to the journal's Software and High-Performance Computing section July 17, 2017; accepted for publication (in revised form) September 12, 2018; published electronically November 6, 2018.

http://www.siam.org/journals/sisc/40-6/M113886.html

Funding: The first author's research was supported by the "Consejo Nacional de Ciencia y Tecnología (CONACyT, México)" under grant 231588290790.

${ }^{\dagger}$ Barcelona Supercomputing Center (BSC), Barcelona, 08034 Spain (juan.cajas@bsc.es, guillaume.houzeaux@bsc.es, mariano.vazquez@bsc.es, marta.garcia@bsc.es, eva.casoni@bsc.es, hadrien.calmet@bsc.es, antoni.artigues@bsc.es, ricard.borrell@bsc.es, oriol.lehmkuhl@bsc.es, daniel. pastrana@bsc.es).

${ }^{\ddagger}$ IIIA - CSIC, Bellaterra, 08193 Spain.

$\S$ Vortex Bladeless, Madrid, 28232 Spain (david@vortexbladeless.com).

ฯIQS School of Engineering, Universitat Llull, Barcelona, 08017 Spain (ponscramon@gmail.com, jordi.martorell@iqs.url.edu).

C677 
state of the art in FSI problems can be found in $[4,37]$ and references therein. In order to be able to develop numerical tools able to cope with the complexity of both problems, the correct modeling of the physical coupling is a crucial aspect. In the development of these numerical tools, the first choice to be made is between two main paths to cast the problem. The first one is known as the monolithic approach, where the full coupled problem is discretized in a single matrix and advanced in time as a global system. This approach is robust and can provide very accurate results. However, it doesn't allow the use of pre-existing independent solvers for the solid and the fluid, and a large amount of recoding is usually required in its development. Some examples of the monolithic approach can be found in [27, 23, 13, 2, 21]. The second one is known as the partitioned or staggered approach, where the problem is solved in separated domains, and the solution of the full problem is achieved by the correct use of the physical coupling conditions. This approach allows one to use pre-existing solvers for the fluid and the solid problems, including black-box commercial solvers. However, it is prone to present robustness problems.

In both cases, to achieve a correct numerical simulation of FSI problems, it is of prime importance to be able to track the solid-fluid interface where the physical coupling conditions are to be imposed. Different means can be used to this end, like the interface capturing techniques, where the fluid and the solid equations are solved in a fixed spatial domain, and an interface function marking its position has to be calculated. Another mean used are the interface-tracking techniques, where the grid moves to track the interface position, and a mesh movement equation is used. For example, one of the most widely extended approaches of this type is obtained through the arbitrary Lagrangian Eulerian (ALE) formulation, where the fluid equations are written in a moving Eulerian reference system, which translates in a moving grid that tracks the interface in the numerical formulation. The movement of the grid points is usually modeled through a partial differential equation. Some examples of the use of the ALE formulation are in [33, 29]. And an interesting example of the use of radial basis functions (RBFs) can be seen in [12].

Numerous studies of FSI problems using the partitioned approach have been developed through the years, identifying the most defiant problems of the formulation and proposing diverse solutions in order to obtain robust and stable simulation tools; see, for example, [44, 39, 45, 46, 31]. In the previous contributions, besides the general theoretical framework for FSI, two main difficulties are pointed out. The first is the fulfilment of the geometric conservation law, which is a consistency criterion obtained from the statement that an arbitrary movement of the mesh must not alter a constant flow. The studies reveal that if this criterion is not considered, unstable schemes may be obtained [30]. The second is the presence of the added mass effect, which is an instability arising for incompressible fluid flow and that depends on the fluid and the solid densities, and can lead to unstable staggered schemes [18, 8].

In this paper, a framework developed for massively parallel simulation of FSI problems in a multicode partitioned approach, taking into account all the aspects mentioned above, is presented. The organization of the paper is as follows. In section 2 , the problem is posed and the coupling is algebraically represented by means of a transmission matrix. Also, two different coupling algorithms with different acceleration schemes are revised. Section 3 is devoted to the parallel implementation, where the main points of interest are the parallel framework needed to be able to transfer data among the codes, the parallel location of the exchange surface (the fluid-solid interface), and the communication strategy. In section 4, three representative cases of different areas of interest are solved to showcase the versatility of the proposed 
framework; good agreement compared to the literature and experiments is achieved. Finally, different ways of distributing the MPI processes of the fluid and the solid codes through the available supercomputing nodes are presented and their efficiencies compared in section 5 .

1.1. Multicode coupling for parallel applications. Multicode coupling poses different computational challenges, namely the need to exchange data between applications that can be written in different programming languages, normally with different mathematical formulations and data structures. Also, new synchronization points appear and a new level of load balance is created. Thus having good configurations for each component of the multiphysics simulation doesn't guarantee a good performance from the coupled problem.

The intercode communication issue can be addressed using a library approach (see, for example, $[5,34]$ ), where a communication library is used as a bridge between the two codes. In this case, the data structures of the coupled applications are adapted to the data structures of the exchange library, and the communication is performed through the functions of the latter. This approach provides very flexible schemes and has relatively low implementation cost; moreover, it is particularly powerful when different instances of different codes are coupled. On the other hand, it is possible to use an integrated approach, where the intercode communication functions are included as part of the code. This approach is advantageous when two different instances of the same code are to be coupled because the data structures don't have to be modified and the communications functions can be designed in order to fully exploit the already existing data structures. The drawback is that it doesn't provide flexibility for coupling with different codes. In the present paper, this scheme is chosen but the concepts and implementation strategies are general enough to develop both approaches.

No matter which strategy is used to communicate the parallel applications, it is of prime importance that the data exchange between the codes be performed in parallel and in an efficient way, avoiding bottlenecks. This is mandatory in order to obtain the lowest impact possible in the performance of the resultant FSI solver. For this purpose, point-to point communications are proposed, involving only the parallel process relevant to the coupling.

When it comes to CFD or CSD, besides the computational challenges, there also exist formulation issues that have to be taken into account. Different codes will usually use different discretization methods and algorithms; consequently, we use an "indirect" exchange methodology, which means that each code will have to process the received values in order to be consistent with its own formulation.

\section{Fluid-structure interaction.}

2.1. Governing equations. The governing equations for incompressible fluid flow are to be coupled with the Euler equations for solid dynamics. For the incompressible fluid, the Navier-Stokes equations are

$$
\begin{gathered}
\rho_{f} \frac{\partial \boldsymbol{u}_{f}}{\partial t}+\rho_{f}\left(\boldsymbol{u}_{f} \cdot \nabla\right) \boldsymbol{u}_{f}-\nabla \cdot\left[2 \mu_{f} \varepsilon\left(\boldsymbol{u}_{f}\right)\right]+\nabla p=\rho_{f} \boldsymbol{f}, \\
\nabla \cdot \boldsymbol{u}_{f}=0
\end{gathered}
$$

where $\boldsymbol{u}_{f}$ is the fluid velocity field, $\rho_{f}$ is the fluid density, $\mu_{f}$ is the fluid viscosity, $p$ is the pressure, and $\varepsilon$ is the velocity strain rate $\varepsilon=\frac{1}{2}\left(\nabla \boldsymbol{u}_{f}+\nabla \boldsymbol{u}_{f}^{t}\right)$. This system has to be solved with appropriate boundary and initial conditions. 
For the deformable solid, we have

$$
\rho_{s} \frac{\partial \boldsymbol{d}_{s}}{\partial t}=\nabla \cdot \boldsymbol{P}+\boldsymbol{b}
$$

where $\rho_{s}$ is the solid density, $\boldsymbol{d}_{s}$ is the displacement field of the solid, $\boldsymbol{P}$ is the first Piola-Kirchhoff stress tensor, and $\boldsymbol{b}$ represent the body forces. All quantities and derivatives are defined in the reference configuration (total Lagrangian formulation). This equation also has to be provided with the corresponding boundary and initial conditions.

At the interface, the coupling conditions to be imposed are the dynamic and the kinematic continuity; in the continuum level with the usual no-slip condition, this means

$$
\begin{aligned}
\boldsymbol{d}_{f} & =\boldsymbol{d}_{s}, \\
\boldsymbol{t}_{f} & =-\boldsymbol{t}_{s}, \\
\boldsymbol{u}_{f} & =\frac{\partial \boldsymbol{d}_{f}}{\partial t},
\end{aligned}
$$

where $\boldsymbol{d}_{f}$ is the displacement of the solid interface inside the fluid and $\boldsymbol{t}_{f}$ and $\boldsymbol{t}_{s}$ are the tractions exerted on the interface by the fluid and the solid, respectively.

2.2. Interface tracking for the fluid flow problem. In the present contribution, the arbitrary Lagrangian Eulerian (ALE) formulation is employed. In this case, the fluid conservation laws are written in a moving Eulerian domain and the governing equations are written in this frame of reference. This formulation is widely used $[16,29,10,38]$; therefore, the steps to obtain the governing equations are not repeated herein. The resulting equations are

$$
\begin{gathered}
\rho_{f} \frac{\partial \boldsymbol{u}_{f}}{\partial t}+\rho_{f}\left[\left(\boldsymbol{u}_{f}-\boldsymbol{u}_{m}\right) \cdot \nabla\right] \boldsymbol{u}_{f}-\nabla \cdot\left[2 \mu_{f} \boldsymbol{\varepsilon}\left(\boldsymbol{u}_{f}\right)\right]+\nabla p=\rho_{f} \boldsymbol{f} \\
\nabla \cdot \boldsymbol{u}_{f}=0
\end{gathered}
$$

where $\boldsymbol{u}_{m}$ represents the domain velocity. In our implementation, $\boldsymbol{u}_{m}$ is obtained from the domain displacement, $\boldsymbol{d}_{m}$, computed as the solution of a diffusion equation of the form

$$
\nabla \cdot\left[c_{m} \nabla \boldsymbol{d}_{m}\right]=0
$$

where $c_{m}$ is a diffusion coefficient. At the discrete level, $\boldsymbol{d}_{m}$ is the node displacement and $c_{m}$ is computed elementwise in order to control the stiffness of the elements. In this work, $c_{m}$ is a discontinuous function computed as

$$
c_{m}=A R / V
$$

with $A R$ the aspect ratio and $V$ the volume of the element. In this way, small elements and elements with large aspect ratio will be "stiffer," which in practice is found to be useful in order to preserve the boundary layer elements.

2.3. Some words on the added mass effect. As explained in $[18,8]$, the added mass effect occurs when an incompressible FSI problem is solved by means of a staggered algorithm. This is an instability coming from the coupling algorithm that destroys the convergence of the staggered scheme. The effect is critical when the ratio of the solid and the fluid densities is close to unity and it is reported to become stronger as the time step decreases. 
The added mass effect can be understood as follows: when the solid displaces, the domain of the fluid moves regardless of the incompressibility condition of the fluid; this creates an unphysical configuration that gives place to a large pressure gradient that opposes the movement, and the resulting forces coming from this pressure gradient displace the solid in the opposite direction, creating the corresponding pressure gradient in the opposite way. If this change is not smooth enough, the simulation blows up in few iterations. In order to treat this instability, different underrelaxation schemes have been tested with satisfactory results. In the present contribution, Aitken's underrelaxation factor as presented in [10] is used.

2.4. The matrix discrete problem. In the quest to obtain a coupling strategy independent of the discretization used, it is convenient to rewrite the full problem in algebraic matrix form. Let us consider the algebraic form of the discretized problems independently. We will have three matrices coming from the fluid flow problem, the mesh movement (e.g., represented by a Poisson equation), and the solid mechanics problem. Therefore, at time $t^{n+1}$, and given a linearization iteration, we have the three following decoupled systems:

$$
\mathbf{A}_{f} \mathbf{u}_{f}=\mathbf{b}_{f}, \quad \mathbf{A}_{m} \mathbf{d}_{m}=\mathbf{b}_{m}, \quad \mathbf{A}_{s} \mathbf{d}_{s}=\mathbf{b}_{s},
$$

where $\mathbf{A}_{f}, \mathbf{A}_{m}$, and $\mathbf{A}_{s}$ are the matrices of the fluid flow problem, the mesh displacement problem, and the solid mechanics problem, respectively. The coefficients of these matrices will normally depend on the problem's unknowns. The velocity of the fluid is $\mathbf{u}_{f}$, and the mesh displacement is calculated with reference to the previous time step configuration and is denoted by $\mathbf{d}_{m}$, while the solid displacement is $\mathbf{d}_{s}$ and is calculated with respect to the original configuration. It is important to keep in mind the different reference frames for $\mathbf{d}_{m}$ and $\mathbf{d}_{s}$.

Let us assume the fluid and the solid meshes coincide on their interface, referred to as the "wet surface," and define the nodes where this interface lies as the "wet nodes." The rest of the nodes will be identified as "dry nodes." It is possible to separate wet and dry nodes using a node renumbering; by doing so, it is possible to write

$$
\left(\begin{array}{cc|cc|cc}
\mathbf{A}_{f f} & \mathbf{A}_{f \Gamma_{f}} & 0 & 0 & 0 & 0 \\
\mathbf{A}_{\Gamma_{f} f} & \mathbf{A}_{\Gamma_{f} \Gamma_{f}} & 0 & 0 & 0 & 0 \\
\hline 0 & 0 & \mathbf{A}_{m m} & \mathbf{A}_{m \Gamma_{m}} & 0 & 0 \\
0 & 0 & \mathbf{A}_{\Gamma_{m} m} & \mathbf{A}_{\Gamma_{m} \Gamma_{m}} & 0 & 0 \\
\hline 0 & 0 & 0 & 0 & \mathbf{A}_{s s} & \mathbf{A}_{s \Gamma_{s}} \\
0 & 0 & 0 & 0 & \mathbf{A}_{\Gamma_{s} s} & \mathbf{A}_{\Gamma_{s} \Gamma_{s}}
\end{array}\right)\left(\begin{array}{l}
\mathbf{u}_{f} \\
\mathbf{u}_{\Gamma_{f}} \\
\hline \mathbf{d}_{m} \\
\mathbf{d}_{\Gamma_{m}} \\
\hline \mathbf{d}_{s} \\
\mathbf{d}_{\Gamma_{s}}
\end{array}\right)=\left(\begin{array}{l}
\mathbf{b}_{f} \\
\mathbf{b}_{\Gamma_{f}} \\
\hline \mathbf{b}_{m} \\
\mathbf{b}_{\Gamma_{m}} \\
\hline \mathbf{b}_{s} \\
\mathbf{b}_{\Gamma_{s}}
\end{array}\right) .
$$

Here, $\mathbf{A}_{i i}$ is the matrix that corresponds to the dry nodes denoted by $\mathbf{u}_{i}$, the matrix that corresponds to the wet nodes $\mathbf{u}_{\Gamma_{i}}$ is $\mathbf{A}_{\Gamma_{i} \Gamma_{i}}$, and the matrices that relate dry and the wet nodes are $\mathbf{A}_{i \Gamma_{i}}$ and $\mathbf{A}_{\Gamma_{i} i}$. The coupling conditions dictate that the displacement of the fluid mesh and the solid displacement are the same at the wet surface, so

$$
\mathbf{d}_{\Gamma_{m}}=\mathbf{d}_{\Gamma_{s}}-\mathbf{d}_{\Gamma_{s}}\left(t_{n}\right),
$$

where $\mathbf{d}_{\Gamma_{s}}\left(t_{n}\right)$ is the solid displacement at the wet surface in the previous time step. Also, if the wet surface is a nonslipping surface, the fluid velocity at this point will be the velocity of this surface; at first order, we can write

$$
\mathbf{u}_{\Gamma_{f}}=\frac{\mathbf{d}_{\Gamma_{m}}}{\delta t} .
$$


Now, the force exerted by the fluid over the solid is given by the residual of the momentum equation at the wet surface [17],

$$
\mathbf{r}_{\Gamma_{f}}=\mathbf{b}_{\Gamma_{f}}-\mathbf{A}_{\Gamma f} \mathbf{u}_{f}-\mathbf{A}_{\Gamma_{f} \Gamma_{f}} \mathbf{u}_{\Gamma_{f}}
$$

and must be added to the wet nodes of the solid problem. Note that this can be done if the units of both the fluid and the solid equations are the same.

The coupled problem is then written as follows:

$$
\left(\begin{array}{cc|cc|cc}
\mathbf{A}_{f f} & \mathbf{A}_{f \Gamma_{f}} & 0 & 0 & 0 & 0 \\
0 & \mathbf{I} & 0 & -\mathbf{I} / \delta t & 0 & 0 \\
\hline 0 & 0 & \mathbf{A}_{m m} & \mathbf{A}_{m \Gamma_{m}} & 0 & 0 \\
0 & 0 & 0 & \mathbf{I} & 0 & -\mathbf{I} \\
\hline 0 & 0 & 0 & 0 & \mathbf{A}_{s s} & \mathbf{A}_{s \Gamma_{s}} \\
\mathbf{A}_{\Gamma_{f} f} & \mathbf{A}_{\Gamma_{f} \Gamma_{f}} & 0 & 0 & \mathbf{A}_{\Gamma_{s} s} & \mathbf{A}_{\Gamma_{s} \Gamma_{s}}
\end{array}\right)\left(\begin{array}{l}
\mathbf{u}_{f} \\
\mathbf{u}_{\Gamma_{f}} \\
\mathbf{d}_{m} \\
\mathbf{d}_{\Gamma_{m}} \\
\mathbf{d}_{s} \\
\mathbf{d}_{\Gamma_{s}}
\end{array}\right)=\left(\begin{array}{c}
\mathbf{b}_{f} \\
0 \\
\frac{\mathbf{b}_{m}}{-\mathbf{d}_{\Gamma_{s}}\left(t_{n}\right)} \\
\hline \mathbf{b}_{s} \\
\mathbf{b}_{\Gamma_{f}}+\mathbf{b}_{\Gamma_{s}}
\end{array}\right) .
$$

Note that matrices $\mathbf{A}_{\Gamma_{m} m}=0$ and $\mathbf{A}_{\Gamma_{m} \Gamma_{m}}=I$ in order to impose the correct boundary conditions for the mesh movement problem. Also, it is important to remember that the matrices $\mathbf{A}_{\Gamma_{i} \Gamma_{i}}, \mathbf{A}_{i \Gamma_{i}}$, and $\mathbf{A}_{\Gamma_{i} i}$ will normally be functions of the unknowns $\mathbf{u}_{i}$, and in order to linearize the problem the values of these quantities from previous iterations can be used, which can be useful when defining coupling strategies. In addition, the coupling of the mesh displacement with the fluid appears also in matrices $\mathbf{A}_{f f}$ and $\mathbf{A}_{f \Gamma_{f}}$, through the mesh velocity computed as $\mathbf{d}_{m} / \delta t$ and $\mathbf{d}_{\Gamma_{m}} / \delta t$.

2.5. Coupling strategy. The coupling strategy presented here is based on an iterative staggered multicode approach, and the individual systems to solve can be obtained from (11). Casting the coupling terms to the right-hand side, we get

$$
\left(\begin{array}{cccc:cc}
\mathbf{A}_{f f} & \mathbf{A}_{f \Gamma_{f}} & 0 & 0 & 0 & 0 \\
0 & \mathbf{I} & 0 & 0 & 0 & 0 \\
0 & 0 & \mathbf{A}_{m m} & \mathbf{A}_{m \Gamma_{m}} & 0 & 0 \\
0 & 0 & 0 & \mathbf{I} & 0 & 0 \\
\hdashline 0 & 0 & 0 & 0 & \mathbf{A}_{s s} & \mathbf{A}_{s \Gamma_{s}} \\
0 & 0 & 0 & 0 & \mathbf{A}_{\Gamma_{s} s} & \mathbf{A}_{\Gamma_{s} \Gamma_{s}}
\end{array}\right)\left(\begin{array}{c}
\mathbf{u}_{f}^{i+1} \\
\mathbf{u}_{\Gamma_{f}}^{i+1} \\
\mathbf{d}_{m}^{i+1} \\
\mathbf{d}_{\Gamma_{m}}^{i+1} \\
\hdashline \mathbf{d}_{s}^{i+1} \\
\mathbf{d}_{\Gamma_{s}}^{i+1}
\end{array}\right)=\left(\begin{array}{c}
\mathbf{b}_{f} \\
\mathbf{d}_{\Gamma_{m}}^{i+1} / \delta t \\
\mathbf{b}_{m} \\
\mathbf{d}_{\Gamma_{s}}^{i+p}-\mathbf{d}_{\Gamma_{s}}\left(t_{n}\right) \\
\hdashline \mathbf{b}_{s}^{-} \\
\mathbf{b}_{\Gamma_{s}}+\mathbf{r}_{\Gamma_{f}}^{i+q}
\end{array}\right)
$$

where the values of the superindices $p$ and $q$ depend on the choice of the coupling scheme. Note that the fluid and the mesh movement systems are also solved in a staggered way, as the coupling term in the Navier-Stokes equation is casted to the right-hand side (second row) with index $i+1$. This means that the mesh movement is solved before the fluid equations.

In the present contribution, two different instances of the HPC code Alya, which is described in section 3, are used to solve the FSI problem. The first one solves the fluid flow problem together with the fluid mesh movement, and the second one the solid mechanics problem, as indicated by the block separation with dotted lines in (12). From this equation, it is also clear that the quantities to be exchanged between the codes are $\mathbf{d}_{\Gamma_{s}}^{i+p}-\mathbf{d}_{\Gamma_{s}}\left(t_{n}\right)$ from the solid code to the fluid code and $\mathbf{r}_{\Gamma_{f}}^{i+q}$ from the fluid code to the solid code. It is important to be noticed that the matrix in (12) allows one to solve each block independently and use different iterative schemes to perform the coupling on the right-hand side; there exist several options, in general, and they are variations of the two approaches presented below. 
2.5.1. Gauss-Seidel staggered coupling scheme. In this scheme, $p=0$ and $q=1$, and in a given time step, the work-flow to get the displacements, velocity, and pressure at iteration $i+1$ from those of iteration $i$ is outlined in Algorithm 1.

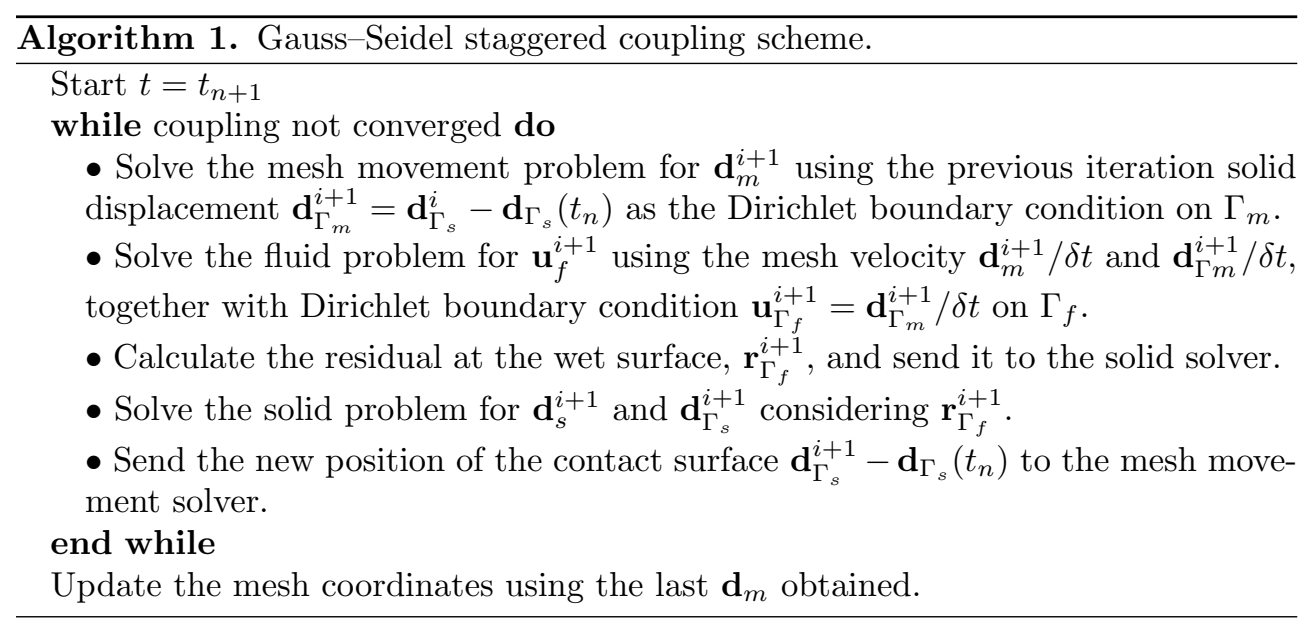

In this case, the solutions of the mesh movement/fluid problems and the solid problem cannot be overlapped because the residual of the current iteration $\mathbf{r}_{\Gamma_{f}}^{i+1}$ is required by the solid solver. Also, given that the boundary condition for the mesh velocity is referred to the previous time step configuration of the solid body $\mathbf{d}_{\Gamma_{s}}\left(t_{n}\right)$, the mesh of the fluid domain must not be moved until the coupling cycle has converged.

2.5.2. Jacobi staggered coupling scheme. In this scheme, $p=q=0$; this means that the residual used in the solid solver comes from the previous iteration and the solutions of both the system's fluid/mesh movement and the solid can be carried out at the same time; the work-flow to get the values of iteration $i+1$ from those of iteration $i$ in a given time step is shown in Algorithm 2. As before, the boundary condition for the mesh velocity is referred to the previous time step configuration of the solid body $\mathbf{d}_{\Gamma_{s}}\left(t_{n}\right)$, and thus the mesh of the fluid domain is only moved when the coupling cycle has converged.

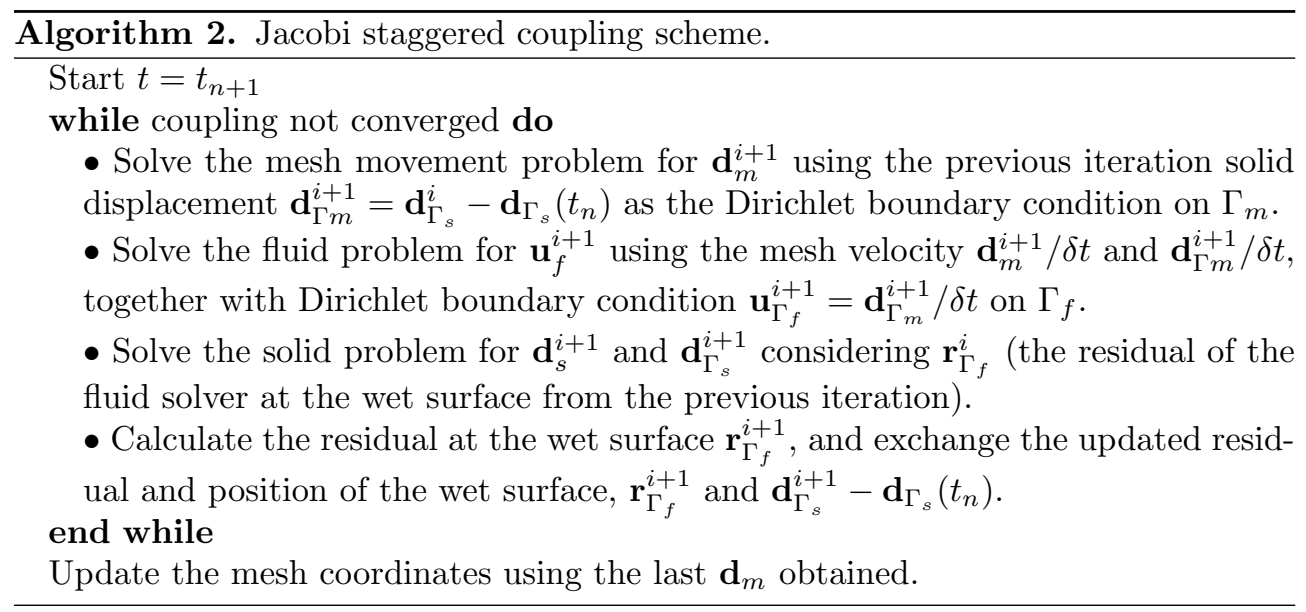

Also, it is pointed out that in the present contribution, a constant underrelaxation 
or Aitken's underrelaxation factor as presented in [10] is used when convergence issues are present.

3. Parallel implementation. The code to be used to perform the numerical simulations of FSI problems is Alya, which is an HPC multiphysics code developed at the Barcelona Supercomputing Center and has been used in different fluid mechanics problems $[6,22,11,1]$ and solid mechanics problems [42]. It is is based on a finite element formulation and parallelized using a hybrid MPI+OpenMP strategy. This code is structured using a modular architecture divided in kernel and modules. The kernel contains the facilities required to solve sets of discretized partial differential equations, while the modules provide the physical description of a given problem. There are modules to handle several different physical problems, and in the present contribution, the modules for the fluid flow problem to be used are Nastin and Alefor. Nastin has the physical description of the Navier-Stokes equations for incompressible fluids, and Alefor provides the facility to deform the mesh of Nastin. For the solid mechanics problem, the module Solidz is used, which is described in [7].

3.1. Multicode coupling parallel communicators. In order to solve the FSI problem in a staggered multicode coupling approach, first of all, it is necessary to enable communications between the involved codes in the simulation. In our implementation, this is done through MPI messages, but care must be taken to not affect the internal code parallelization. In our case, this parallelization is based on a masterworker approach that relies mainly on an MPI parallel processes separation that has proven to scale efficiently in different supercomputers [43].

To be able to communicate with other applications while keeping intact the communication scheme of the code, two extra MPI communicator levels were added on top of the existing layers. These extra layers are particularly convenient because they allow the code to send MPI messages to other instances of the same code or other different applications. In practice, the MPI_COMM_WORLD communicator is replaced by the communicator called PAR_COMM_UNIVERSE, and successive calls to the MPI_COMM_SPLIT() function are used to define the usual Alya communicators.

The MPI communicators hierarchy within the code employed is shown in Figure 1. The initial MPI_COMM_SPLIT() function is applied using the name of the applications inside the PAR_COMM_UNIVERSE communicator, and each MPI process gets a rank in the PAR_COMM_WORLD communicator (called MY_WORLD_RANK). The split creates two independent communicators: the ALYA_WORLD, where all instances of the Alya code will lay, and the REST_OF_WORLD, where all other parallel applications will be included. Then, if there are multiple instances of the Alya code, a second MPI_COMM_SPLIT (using the name of the case) is performed in the ALYA_WORLD communicator. This will separate the ALYA_WORLD in COMM_CODES communicators, one for each running instance of Alya. With all these communicators, it is already possible to exchange information between different codes, but it is convenient to have a subcommunicator where only the parallel partitions involved in the coupling are present; this communicator is referred to as COMM_COUPLING in Figure 1.

3.2. Parallel definition of the coupling surface. Normally, in an FSI problem, it is not known a priori on which parallel partitions (MPI processes) the wet surface is located. Thus, in order to determine those involved in the multicode coupling, an initial communication phase is needed. In the present implementation, each code locates its participant parallel partitions using the boundary label that designates the wet surface. A parallel partition is selected only if among its nodes the wet 


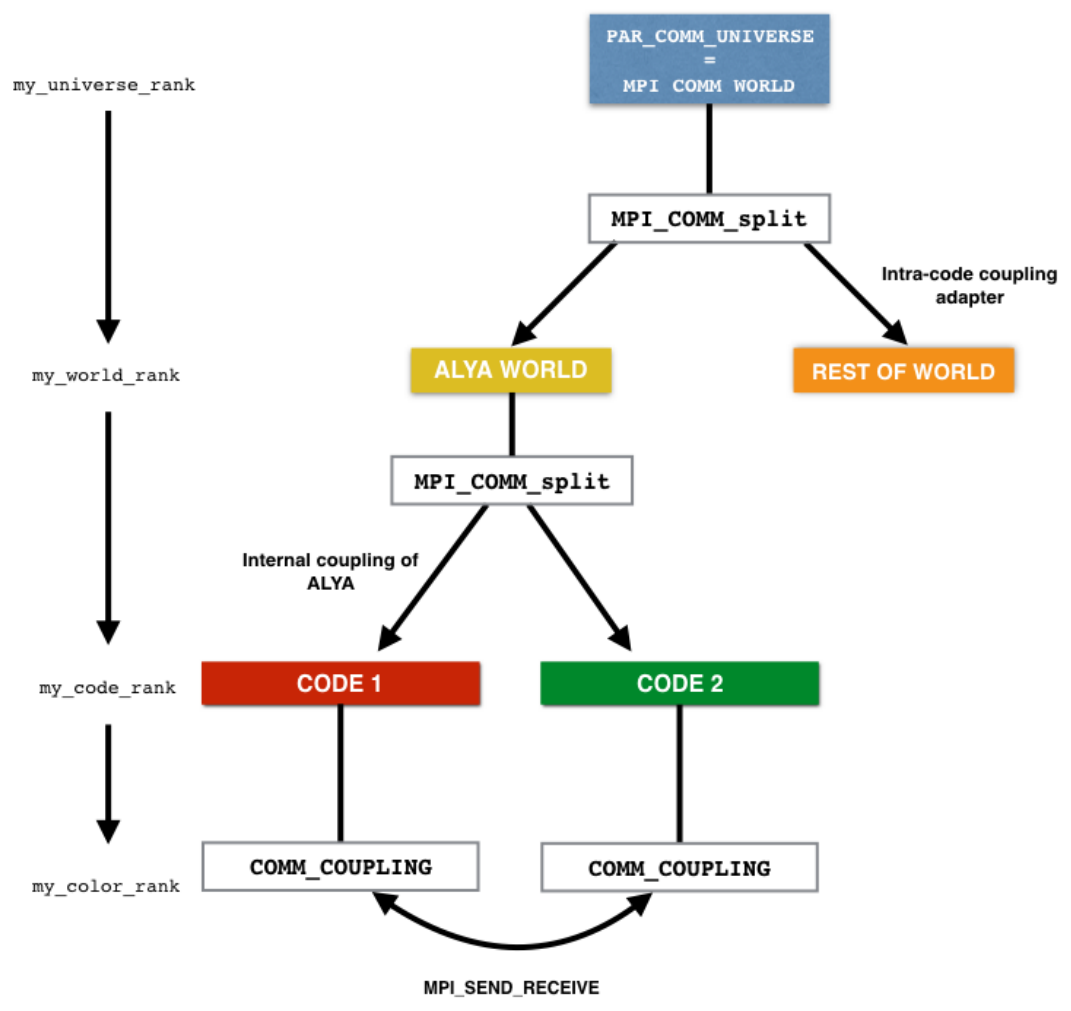

FIG. 1. MPI communicators hierarchy in the Alya code.

surface label is present. Then this parallel partition creates a list of wet nodes with all the nodes that share this label. Once each code has finished this selection, the COMM_COUPLING subcommunicator is created from the ALYA_WORLD communicator, including the selected partitions from the two codes.

After the selection of participant parallel partitions has ended, a search for specific neighbors from the other code is performed. All parallel partitions do the same, so the description will be done from the perspective of one of them. Before going further, it is convenient to introduce some distinctions. We will refer to wet nodes as the nodes of the wet surface that belong to a specific computational domain, while the nodes of the wet surface lying on other parallel partitions will be called test points. From the point of view of a single partition that is involved in the coupling, the steps to follow in order to define its specific neighbors are listed in Algorithm 3.

Once these steps are done, the communications scheme for the coupling is complete. Each parallel partition knows the amount of data it has to send and knows the receivers, and also knows the amount of data it will receive and the senders. With this information, the communication buffers to perform the point-to-point communications required on the multicode coupling can be set up. The described process is schematically shown in Figure 2. Figure 2(a) shows the wet surface on each code. The colors represent different parallel partitions, and the numbers are their ranks in the ALYA_WORLD communicator. Figure 2(b) shows the partitions of each code included in the COMM_COUPLING and its corresponding bounding boxes, and the 


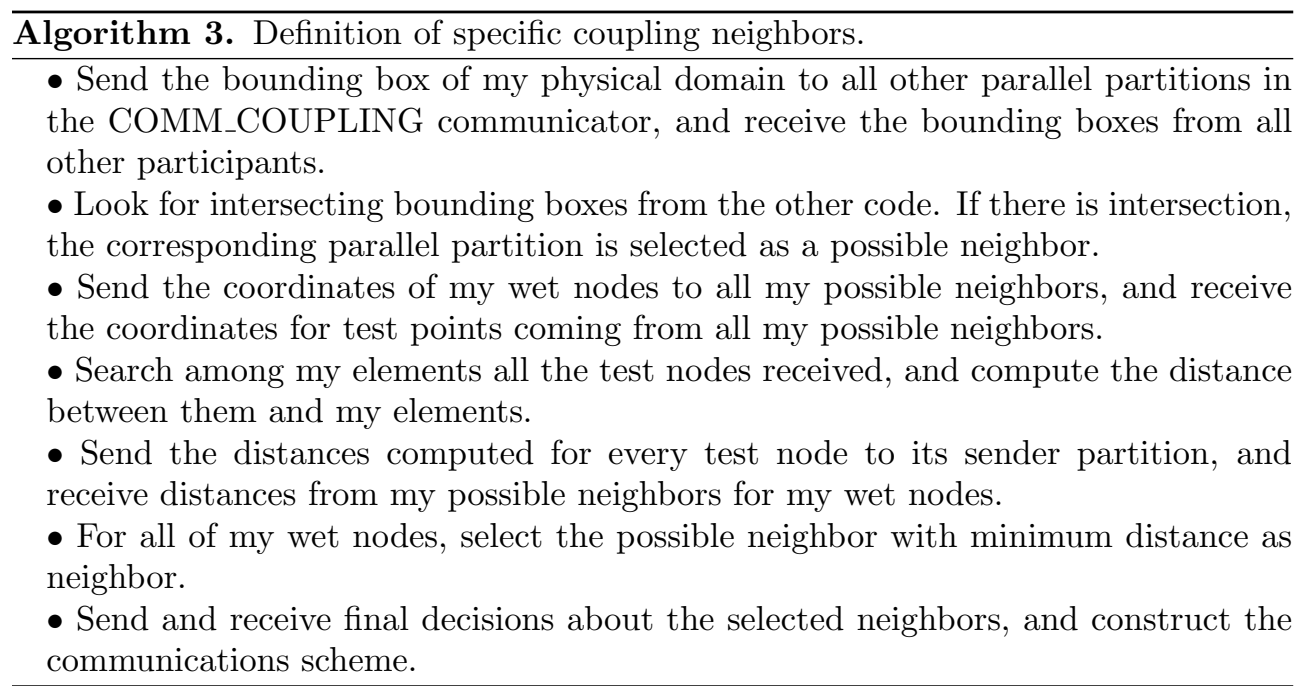

numbers are the ranks of the parallel partitions in the COMM_COUPLING communicator. Figure 2(c) shows the neighbors of partition 1 and the final decision of the wet nodes owners by the colors of the nodes.
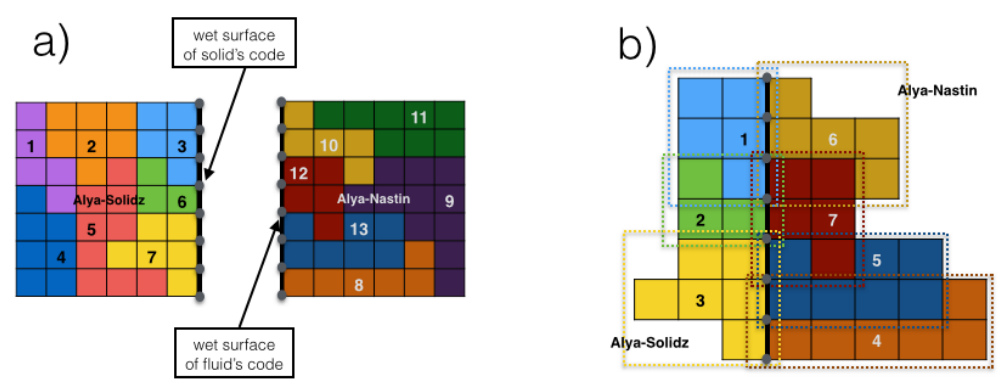

c)

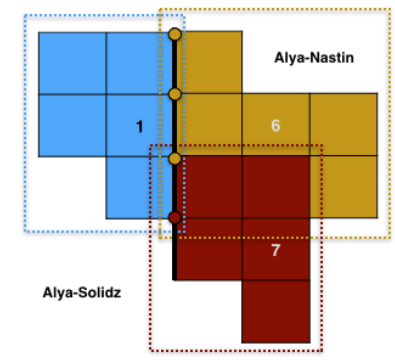

FIG. 2. Schematic representation of the parallel definition of the coupling surface. (a) shows the wet surface on each code. The colors (available online only) represent different parallel partitions, and the numbers are its rank in the ALYA_WORLD communicator. (b) shows the COMM_COUPLING participants and its corresponding bounding boxes; the numbers are the ranks of the parallel partitions in the COMM_COUPLING communicator. (c) shows the neighbors of partition 1 and the final decision of the wet nodes owners by the color of the nodes. Alya-Nastin stands for the fluid code and Alya-Solidz for the solid one. 
For simplicity, the description of the process is limited to the case of conforming meshes; this means that the nodes of the wet surface coincide. In the general case, where nonconforming meshes are treated, interpolation functions or an extra communication step would be needed in order to properly calculate the quantities of interest at the wet nodes. A general description of interpolation techniques is provided in [25].

3.3. Exchange of information. Normally, and in general terms, the work-flow of engineering applications designed to deal with nonlinear equations can be represented as in Figure 3. The preprocessing phase stands for the reading of the input files of the code, memory allocation, communications setup, and data structure construction. The time loop is used to advance in time in transitory problems, and the nonlinear iteration loop solves the nonlinear problem using a linear algebraic solver; inside this loop, the system of algebraic linear equations is assembled (Assemble matrix) and afterwards this system is solved (Solve linear problem). The postprocessing step represents the memory deallocation and the end of the run.

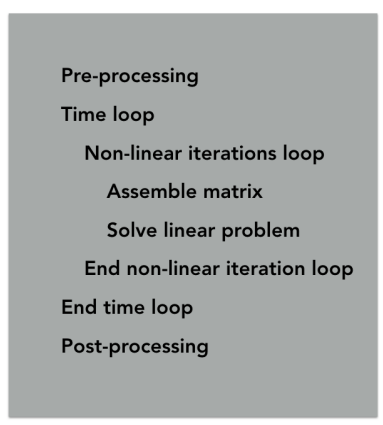

FIG. 3. Common work-flow of an engineering application.

In the present implementation, after the preprocessing step, an initialization of the coupling phase is added, where the parallel definition of the wet surface described is performed, and depending on the coupling strategy, different exchanges of information points can be chosen, as shown in Figure 4, where (a) represents the Gauss-Seidel strategy while (b) represents the Jacobi one.

4. Test cases and numerical results. For the test cases, the following discretization schemes for the fluid and the solid mechanics problems were used: The Navier-Stokes equations were discretized using the stabilized finite element method, with variational multiscale stabilization, which is considered an implicit large eddy simulation method, as explained in [9]. The unknowns of the problem are separated into grid scale components and subgrid scale components with the possibility of tracking the subgrid components in time and space to give more accuracy and stability to the numerical model. The momentum equation is separated from the continuity equation using the Schur complement for the pressure; each equation is solved independently, and the solution of the coupled system is obtained in an iterative way. The time integration scheme used is a backward differentiation formula of second order. The details and validation of the solution strategy are provided in $[26,24]$. For the solid mechanics problem, the Euler equations were discretized using a standard Galerkin method for large deformations with a Newmark time integration scheme; further details can be found in [7]. 


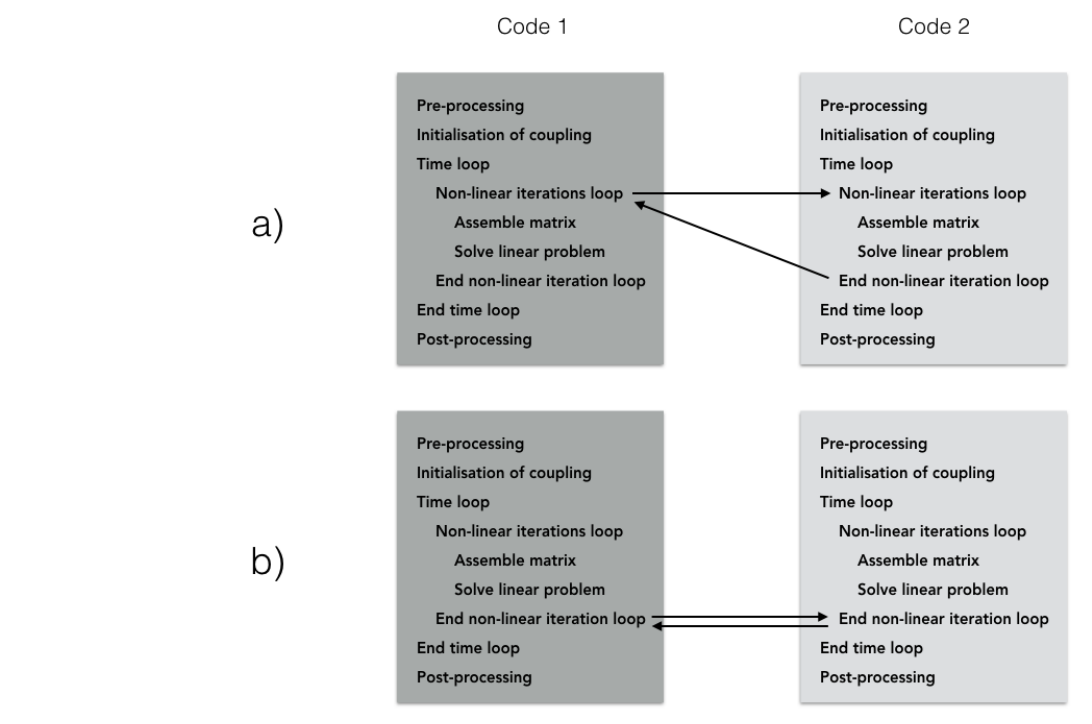

FIG. 4. Exchange of information between the different codes. (a) Gauss-Seidel coupling strategy and (b) Jacobi coupling strategy.

4.1. FSI3 test case. The first test case is a $2 \mathrm{D}$ incompressible laminar flow around a fixed cylinder with an attached elastic Saint Venant-Kirchhoff cantilever. The cylinder is placed in the middle of the flow channel with a small vertical offset. The geometry is depicted in Figure 5. At the left boundary, a parabolic inflow profile is prescribed, while at the right boundary a free outflow condition is imposed. The top and the low wall of the channel, as well as the surface of the cylinder and the cantilever, are considered no-slip boundaries. This configuration was previously proposed in [40] and studied in [41] using a partitioned approach with the PRECICE coupling library $[20]$.

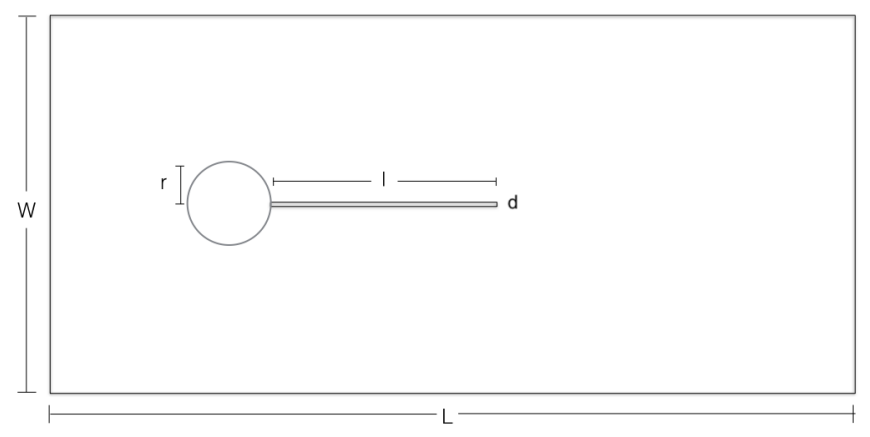

FIG. 5. Schematic representation of the FSI3 test case.

The dimensions of the physical domain (see Figure 5) are $W=0.41 \mathrm{~m}, L=2.5 \mathrm{~m}$, $r=0.05 m, l=0.35 m$, and $d=0.02 m$. The fluid part was discretized using 35,524 triangular elements, while 15,850 triangular elements were used for the solid bar with 


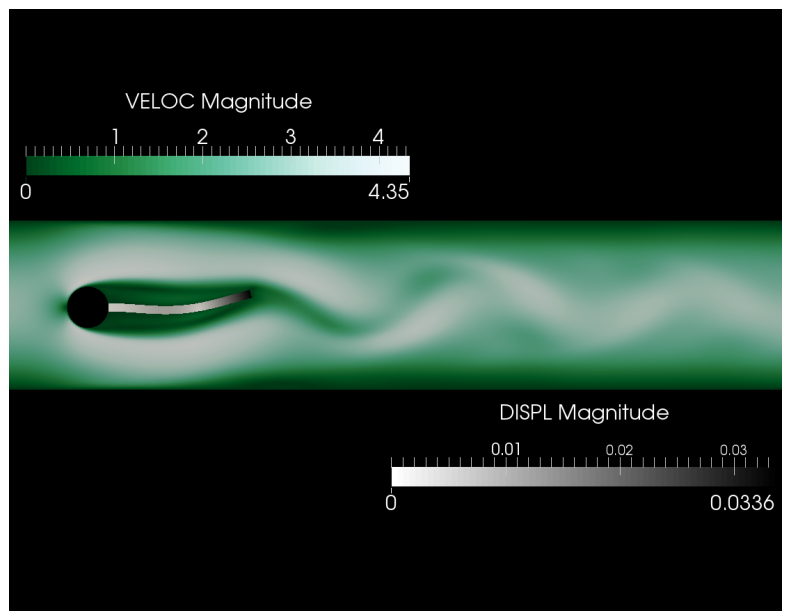

FIG. 6. Typical response of deflection and velocity field of the FSI3 test case.

a time step of $1 \times 10^{-3} \mathrm{~s}$ for both solvers. The density of the fluid is $\rho_{f}=1000 \mathrm{Kg} / \mathrm{m}^{3}$, and its dynamic viscosity is $\mu_{f}=1.0 \mathrm{~Pa}$; the solid has a density of $\rho_{s}=1000 \mathrm{Kg} / \mathrm{m}^{3}$ and is modeled as an isotropic material with Young's modulus of $E=5.6 \times 10^{6} \mathrm{~Pa}$ and Poisson's ratio of $\nu=0.4$. This gives a very challenging added mass effect case, and a strongly Gauss-Seidel coupling algorithm with an Aitken acceleration scheme was used to achieve convergence. It is important to mention that very strict convergence criteria were used in order to get an accurate solution for this case, with an L-2 norm of the residual of the coupling quantities lower than $1 \times 10^{-5}$. On average, with this very strict configuration of the case, a time step required 7.57 coupling iterations and was completed in $17.5 \mathrm{~s}$ using a total of 11 MPI processes, eight for the fluid solver and three for the solid solver. The definition of the coupling surface, the construction of the needed MPI communicators, and the definition of the coupling communications scheme is done in $0.008 \mathrm{~s}$. Typical instantaneous velocity and displacement fields for this case are shown in Figure 6. The complete temporal evolution of the deflection of the tip of the beam is shown in Figure 7 and a detailed view of the last second of the simulation is shown in Figure 8. The results for this case compare satisfactorily to previous studies of different research groups and are included here for validation purposes; a comparison for averaged amplitudes and frequencies in the quasi-periodic state is shown in Table 1.

In order to provide further insight in the convergence and accuracy of the proposed scheme, refined meshes with $277 \mathrm{~K}$ elements for the fluid domain and $64 \mathrm{~K}$ elements for the solid bar were used. The time step was reduced to $5 \times 10^{-4} s$, while the coupling configuration remained the same as before. 36 MPI processes were used in the fluid solver, and 12 were used for the solid one. The setup of the coupling scheme was done in $0.036 \mathrm{~s}$, and a time step required on average 7.47 iterations and was completed in $21.4 \mathrm{~s}$. The difference observed with the previous execution time is due mainly to the difference in the time step used. The results obtained in this mesh are also included in Table 1 and show excellent comparison with the literature.

4.2. Vortex-Bladeless FSI case. The second case presented corresponds to an actual industrial problem in wind energy generation. The device considered comes from the Vortex-Bladeless wind energy generator (http://www.vortexbladeless.com/). 


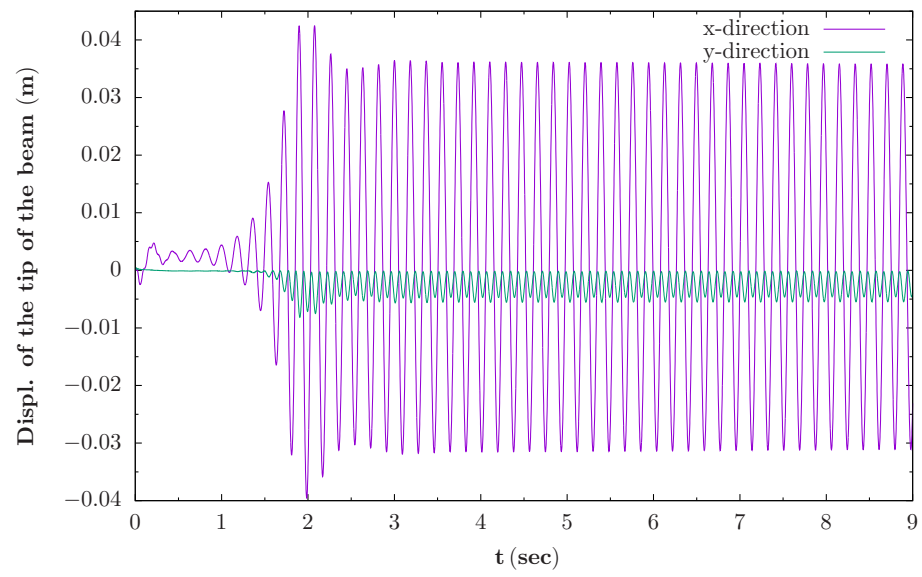

FIG. 7. Deflection of the tip of the beam of the FSI3 test case.

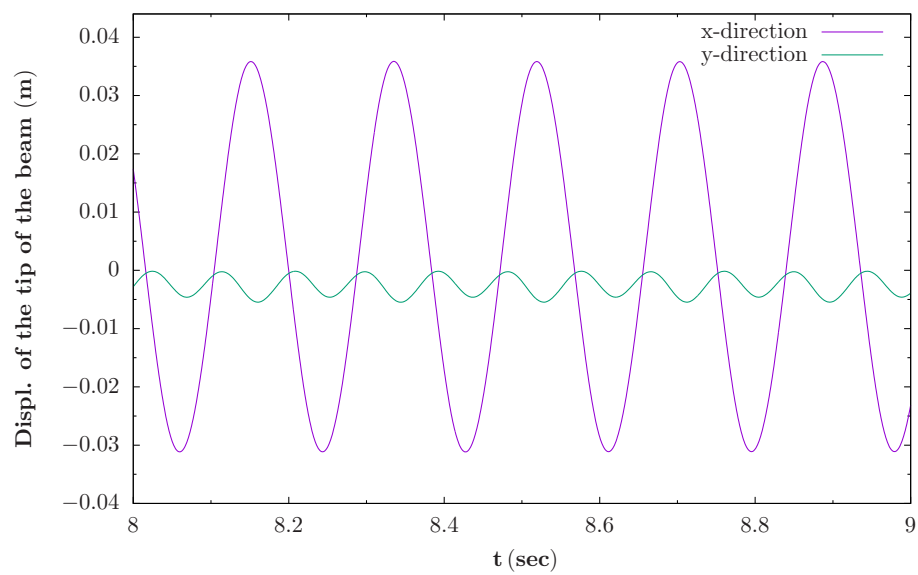

FIG. 8. Detailed view of the deflection of the tip of the beam of the FSI3 test case. The amplitude and frequency of the quasi-periodic final state agree with those reported in the literature.

TABLE 1

Amplitude and frequency comparison for the FSI3 test case. Good agreement between the literature and the present simulation is observed. The results were averaged over the last four cycles of the simulation.

\begin{tabular}{c|c|c}
\hline & Displ. $y(\mathrm{~mm})[$ freq. $H z]$ & Displ. $x(\mathrm{~mm})[$ freq. $H z]$ \\
\hline$[41]$ & $-2.59 \pm 2.42,[10.8]$ & $2.75 \pm 33.32,[5.4]$ \\
{$[40]$} & $-2.69 \pm 2.53,[10.9]$ & $1.48 \pm 34.38,[5.3]$ \\
Simulation coarse mesh & $-2.59 \pm 2.47,[10.8]$ & $2.25 \pm 33.48,[5.4]$ \\
Simulation fine mesh & $-2.80 \pm 2.59,[11.0]$ & $1.76 \pm 34.40,[5.5]$ \\
\hline
\end{tabular}

Vortex Bladeless is a Spanish small medium enterprise (SME) whose objective is to develop a new concept of wind turbine without blades called Vortex. The design aims to take advantage of the vortex induced vibrations (VIV) phenomena to generate energy, aiming to reduce or eliminate many of the existing problems in conventional generators. The simulation considers an experimental device schematically represented in 
Figure 9 inside a wind tunnel with an air velocity of $2.2 \mathrm{~m} / \mathrm{s}$. This represents an FSI problem which is solved in a parallel multicode partitioned approach and is of particular interest given that there exist experimental data recorded by the SME that can be used for comparison and validation.

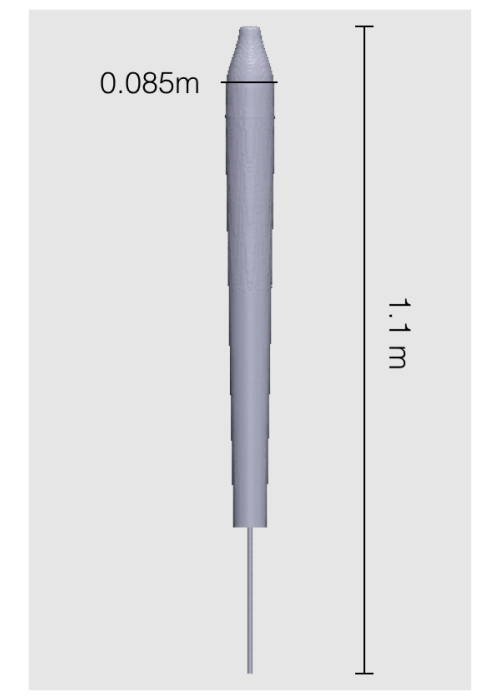

FIG. 9. Schematic representation of the Vortex-Bladeless experimental device.

The computational domain for the solid mechanics problem represents the VortexBladeless experimental device and is considered to be built of two different materials: the more flexible beam in the base with Young's modulus of $E=0.97 \times 10^{11} \mathrm{~Pa}$, Poisson's ratio of $\nu=0.35$, and density $\rho=1365 \mathrm{Kg} / \mathrm{m}^{3}$; and the upper more rigid pole with Young's modulus of $E=3.0 \times 10^{11} \mathrm{~Pa}$ and a mass of $0.091 \mathrm{Kg}$. The geometrical domain is divided in $240 K$ tetrahedral elements, and the simulations were carried out using 32 processors of the MareNostrum III supercomputer. An undamped Newmark temporal scheme with a time step of $2 \times 10^{-3} s$ and a deflated conjugate gradient (DCG) solver for the Euler equations are used.

The computational domain for the fluid flow problem represents a wind tunnel of $20.0 \mathrm{~m}$ of length and a square cross section of $2.0 \mathrm{~m} \times 2.0 \mathrm{~m}$ with an inlet velocity of $2.2 \mathrm{~m} / \mathrm{s}$. The resulting Reynolds number based in the diameter of the pole is $R e=1.1 \times 10^{4}$. The bottom wall and the Vortex-Bladeless device are considered as nonslip surfaces, the side and the top walls are considered slip surfaces, and the outflow is considered a free surface. The computational domain was discretized using $3.3 \mathrm{M}$ elements, most of them tetrahedra. The simulation was run using 357 cores of the MareNostrum III supercomputer. The continuity equation is solved using a DCG solver, while a Generalized Minimal RESidual, GMRES solver is used for the momentum equation. The time step used is $2 \times 10^{-3} s$.

The coupling algorithm is a loosely coupled one. This means that in one time step, the fluid mechanics problem is solved, the forces in the wet surfaces calculated and sent to the solid mechanics code, which after deforming by the effect of these forces sends back the new position of the wet surface and the time step is advanced. This scheme is normally unstable when the densities of the solid body and the fluid are 


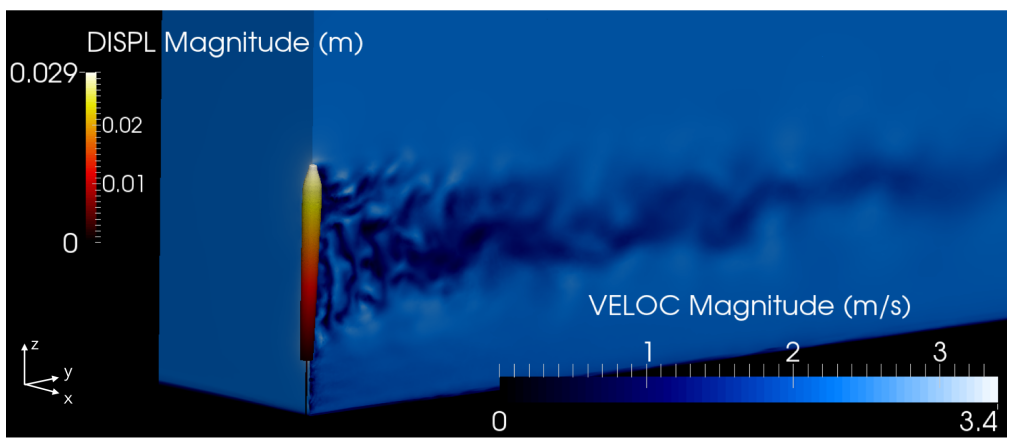

FIG. 10. Typical response of deflection and velocity field of the Vortex-Bladeless FSI case.

similar. However, in the present case, the densities ratio is high and the scheme has proven to be stable. The simulation was started with a fully developed flow around the Vortex-Bladeless device, which was moved slightly from its equilibrium position in order to start with an asymmetrical configuration. The coupling setup for this system took $6.8 s$, and a time step was completed in $13 s$ on average.

A typical response of the system in a given instant of time is depicted in Figure 10, and the oscillatory response of the system is shown in Figure 11, where the displacement of the Vortex-Bladeless device at a height of $z=0.735 \mathrm{~m}$ is plotted. The amplification of the magnitude of the maximum displacement of the Vortex-Bladeless device demonstrates that the lock-in effect is well captured by the FSI simulation and that there is energy transferred from the fluid to the solid. Also, the small oscillations present in the flow direction show that the movement is not confined to a plane. It is remarkable that different low frequencies are present in the overall process, as it can be clearly appreciated from the different peaks and valleys present in the figure.

A more detailed view of the oscillatory response in the last two seconds of the simulation is shown in Figure 12. We can observe that the maximum amplitude of the oscillation doesn't suffer significant changes in this phase and that one single frequency clearly dominates the dynamical behavior of the device. The average maximum amplitude is $A=0.036 \mathrm{~m}$, and the frequency registered is $f=4.8 \mathrm{~Hz}$. Experimental tests performed by the SME for this same device give an average amplitude of $A_{\text {exp }}=0.034 \mathrm{~m}$ and a frequency of $f_{\exp }=5.3 \mathrm{~Hz}$, which is a very good comparison between the tests and the numerical results observed. At present, intensive research is being carried out in order to understand the physics governing this system and evaluate new design proposals.

4.3. Ascending aorta FSI case. The last FSI test case presented in this paper corresponds to the flow of blood inside the ascending aorta. The geometry used in the simulation was obtained from 4D medical images of an ascending aorta aneurysm and was provided by Martorell et. al. [32]. This case is particularly attractive because the geometry is a reconstruction of medical images and it presents a very heavy added mass effect. In the following, the cgs metric system will be used because it is commonly used in the literature of this field and an easier comparison can be done.

The fluid domain is decomposed in $613 \mathrm{~K}$ tetrahedral elements and the simulation run in 48 cpus, the fluid density is set to $\rho_{f}=1.060 \mathrm{~g} / \mathrm{cm}^{3}$, the dynamic viscosity is set to $\mu=3.5 \times 10^{-2} \mathrm{~g} / \mathrm{cm} \mathrm{s}$, and the solid is modeled with an isolinear model with density $\rho_{s}=1 \mathrm{~g} / \mathrm{cm}^{3}$, Young's modulus of $E=4 \times 10^{6} \mathrm{dyn} / \mathrm{cm}^{2}$, and Poisson's ratio 


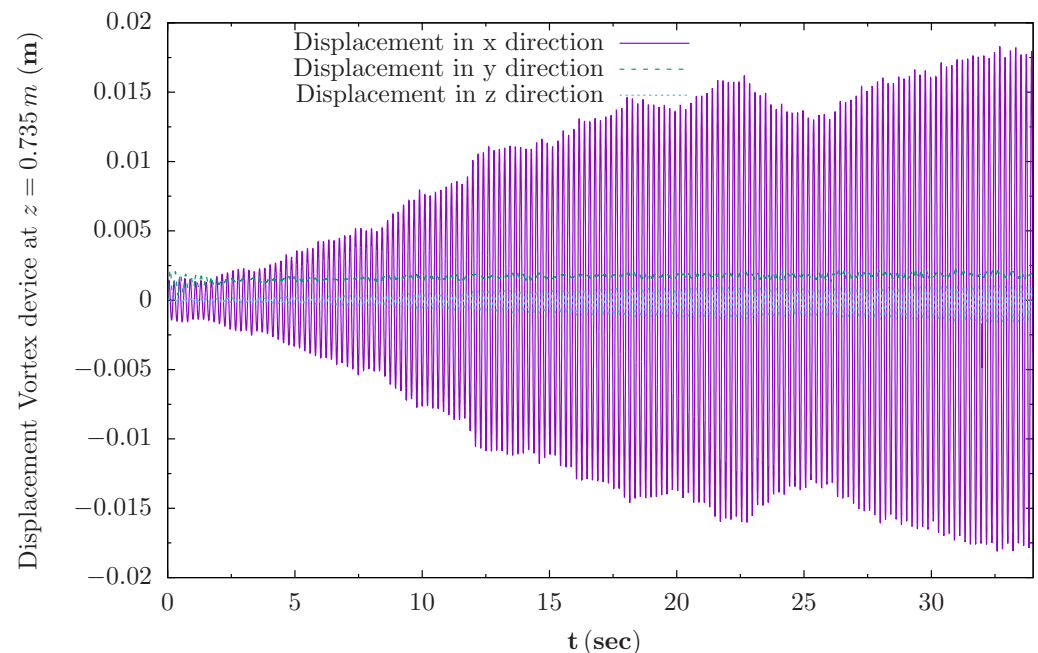

FIG. 11. Displacement of the Vortex-Bladeless device at $z=0.735 \mathrm{~m}$. The amplification of the magnitude of the maximum displacement shows that the lock-in effect is well captured by the FSI simulation. The small oscillations present in the flow direction show that the movement is not confined to a plane and that different low frequencies are present in the overall process.

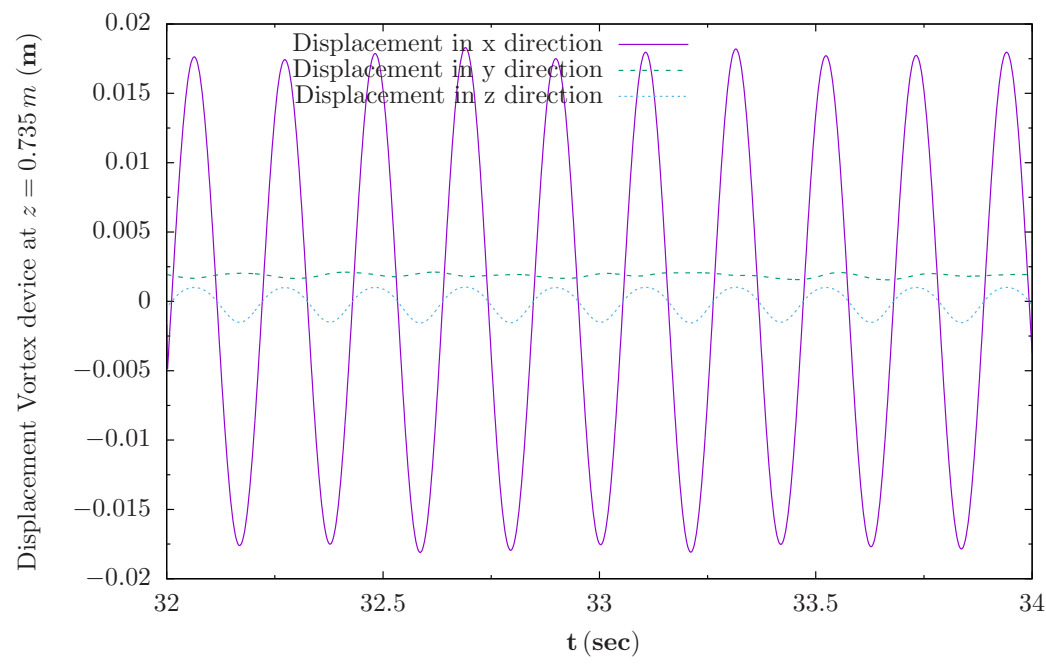

FIG. 12. Detailed view of the oscillatory response in the last two seconds of the simulation. The maximum amplitude of the oscillation doesn't suffer significant changes. The average maximum amplitude is $A=0.036 \mathrm{~m}$, and the frequency registered is $f=4.8 \mathrm{~Hz}$. Fair agreement between the experiments carried out by Vortex Bladeless and the numerical results obtained is observed.

of $\nu=0.4$. The solid domain is discretized using $137 K$ tetrahedral elements, and the simulation was run using 8 cpus.

At the inlet, a plane velocity profile that produces the volumetric flow rate shown in Figure 13 is imposed; this function is chosen in order to reproduce the typical volume inflow to the aorta produced by a heartbeat and was measured during a 


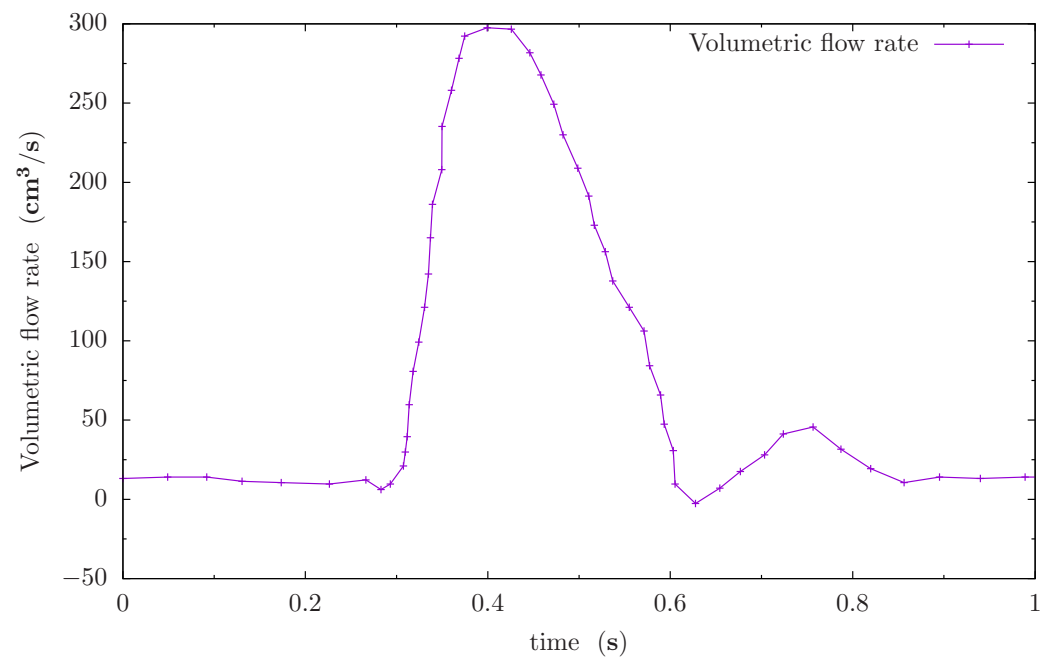

FIG. 13. Volumetric flow rate imposed at the inlet with a plane velocity profile.

doppler study. At the outflow, the pressure is imposed with a function of the form

$$
p=p_{0}+C Q,
$$

where $p_{0}$ is a reference pressure, $C$ is a constant, and $Q$ is the flow rate $\left(\mathrm{cm}^{3} / \mathrm{s}\right)$, to distribute the flow among the upper and lower exits. In the present study, the values used are $p_{0}=800 d y n / \mathrm{cm}^{2}, C_{u p}=50 \mathrm{~g} / \mathrm{cm}^{4} \mathrm{~s}$, and $C_{\text {low }}=1 \mathrm{~g} / \mathrm{cm}^{4} \mathrm{~s}$. The low ends and the three upper branches of the solid body are held fixed, while the rest of the inner and outer walls are allowed to move freely.

The coupling algorithm is a strong coupling Gauss-Seidel method with an Aitken dynamical relaxation parameter. The typical convergence of the coupling algorithm is shown in Figure 14. The L-2 norm of the normalized difference of the results for force and displacement between two successive iterations is used to measure convergence, the tolerance is fixed to $1 \times 10^{-4}$, and convergence is declared when both L-2 norms reach the criteria.

The maximum deformation observed for this case is shown in Figure 15. The present configuration is not intended to simulate a specific patient disease, or a detailed biomechanics case, but to show the capabilities of the numerical tool developed. Nevertheless, the displacements and the velocities obtained are in a reasonable range according to the results found in [3,35]. For this example, the setup of the coupling strategy was done in $0.235 \mathrm{~s}$ and, on average, one time step required 7.29 coupling iterations and was done in $37.6 \mathrm{~s}$.

5. Computational resources usage and efficiency concerns. When dealing with FSI staggered coupled simulations, the robustness of the solver is a major concern and great effort has been devoted to developing efficient coupling algorithms $[15$, 29, 28]. However, the use of the computational resources has not received the same attention and can have a great impact in the overall simulation. For example, a Jacobi algorithm exploits better the computational resources than a Gauss-Seidel algorithm, but at the expense of converging slower, which makes difficult the identification of the best choice. In the following, we offer a strategy to improve the efficiency of the 


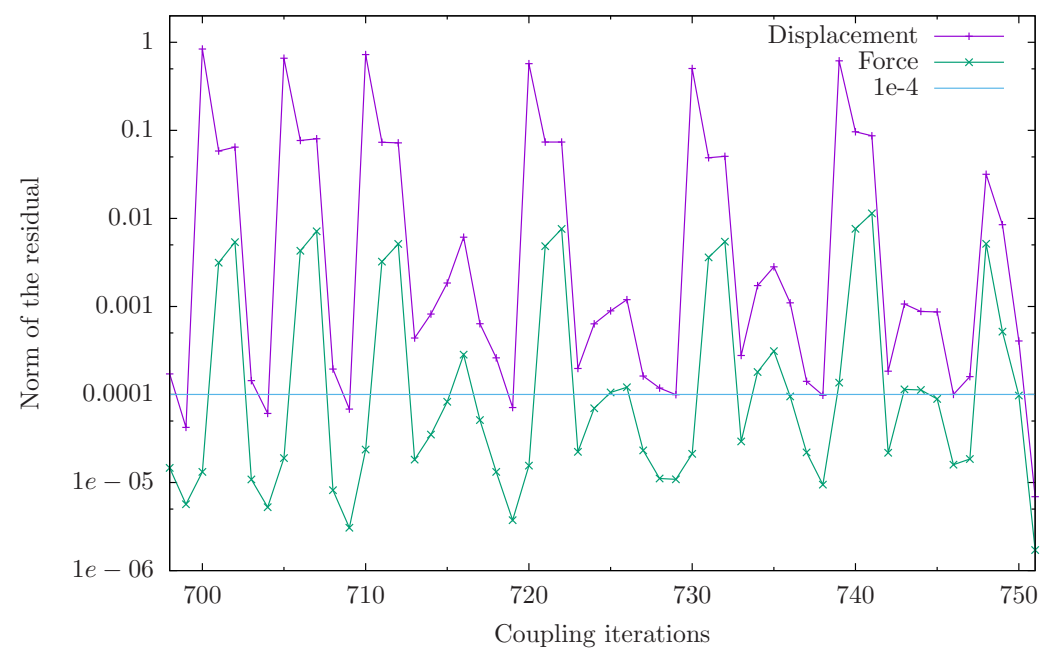

FIG. 14. Convergence of the coupling algorithm for the aorta FSI case using Aitken's relaxation parameter. Convergence is achieved when both $L-2$ norms reach the criteria.

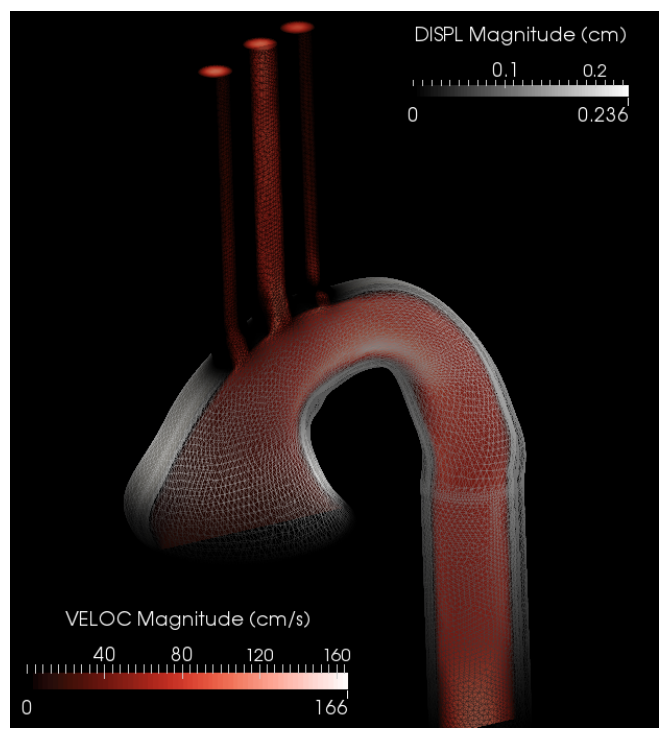

FIG. 15. Displacement and velocity field for the ascending aorta FSI case, with displacements shown in $\mathrm{cm}$ and velocities in $\mathrm{cm} / \mathrm{s}$.

Gauss-Seidel coupling algorithm based on two mechanisms: the overload of the node and the use of the MPI wait mode will be exposed.

In staggered cases, it is very common that when one code is executing, the other one is just waiting for an MPI message, decreasing drastically the efficiency of the parallel execution. We have illustrated this effect by means of traces of the execution of the coupled problem. A trace is a record of the activity of a computer program and is very useful in the design and optimization of HPC tools. In our case, the traces were generated using the profiling tool Extrae [14] and visualized using the performance 
analysis tool Paraver [36]. Figure 16 shows the trace of one time step, including four FSI iterations of the parallel multicode coupled simulation coming from the aorta test case described above. In this illustrative example, we used 32 cores executing 24 MPI parallel processes for the fluid code and eight MPI processes for the solid one. Figure 16(a) illustrates the interleaving between the two MPI communicators solving the fluid (top 24 MPI processes) and the solid (bottom eight MPI processes). Since a staggered approach has been set up, computations of one communicator are overlapped with idle time on the other. Figure16(b) depicts the cycles per microsecond used by the MPI processes; it is observed that with this configuration, there are always processes consuming resources as if they were performing calculations when actually they are just waiting for MPI messages from the other code.

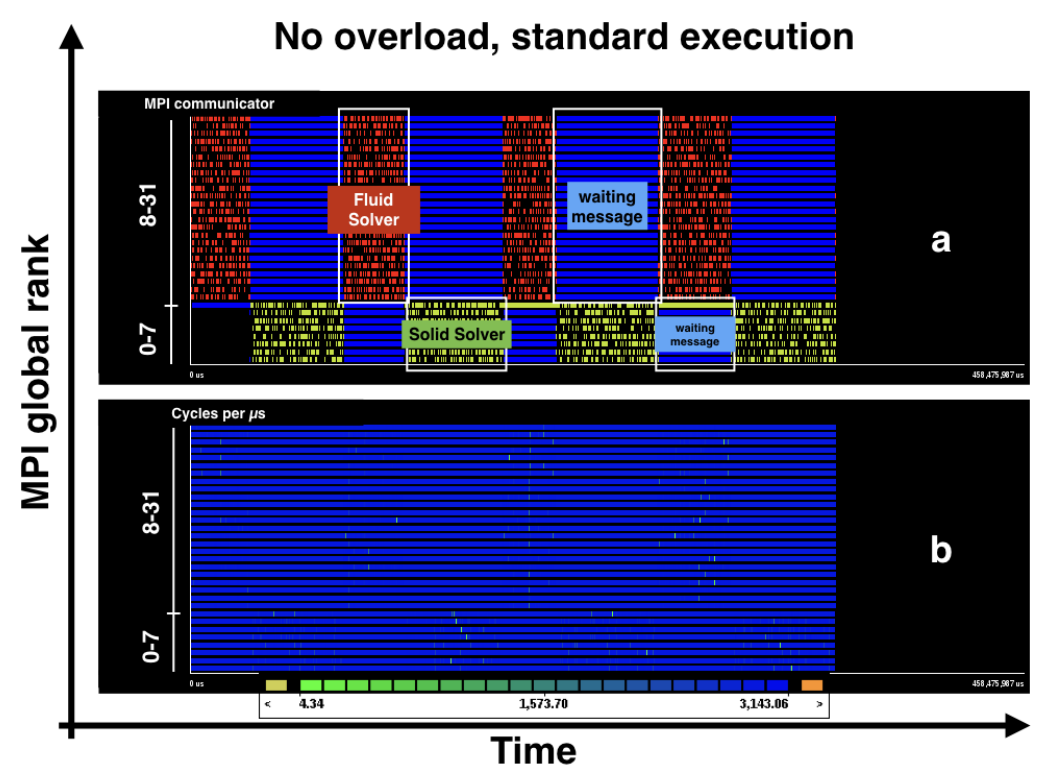

FIG. 16. Trace for an FSI staggered multicode coupled simulation in 32 cores: 24 MPI parallel processes used for the fluid code and eight MPI processes for the solid code. MPI communicator in use (a) and cycles per microsecond used by each core (b).

In order to better use the computational resources, a first natural idea would be to overload the computing cores. As an example, consider the 32 cores of the previous test but executing 48 MPI processes: 32 used for the fluid and 16 for the solid. In this case, there would be 16 overloaded cores that execute one MPI process coming from the fluid code and one coming from the solid code. The idea is that given that the two codes (fluid and solid) never perform computations at the same time, their MPI processes can share the core so that when the fluid MPI process is computing, the solid MPI process is waiting, and vice versa. This strategy was tried, and the results are shown in Figure 17, where the MPI communicator (a) and the cycles per microsecond (b) are shown.

It can be observed that not only was no speed-up achieved, but in fact a drastic increase in the simulation time was obtained. This is because when the MPI processes are waiting for a message, they use a polling mode to check whether it has arrived. The polling mode is a constant sampling of the status of the message that consumes 


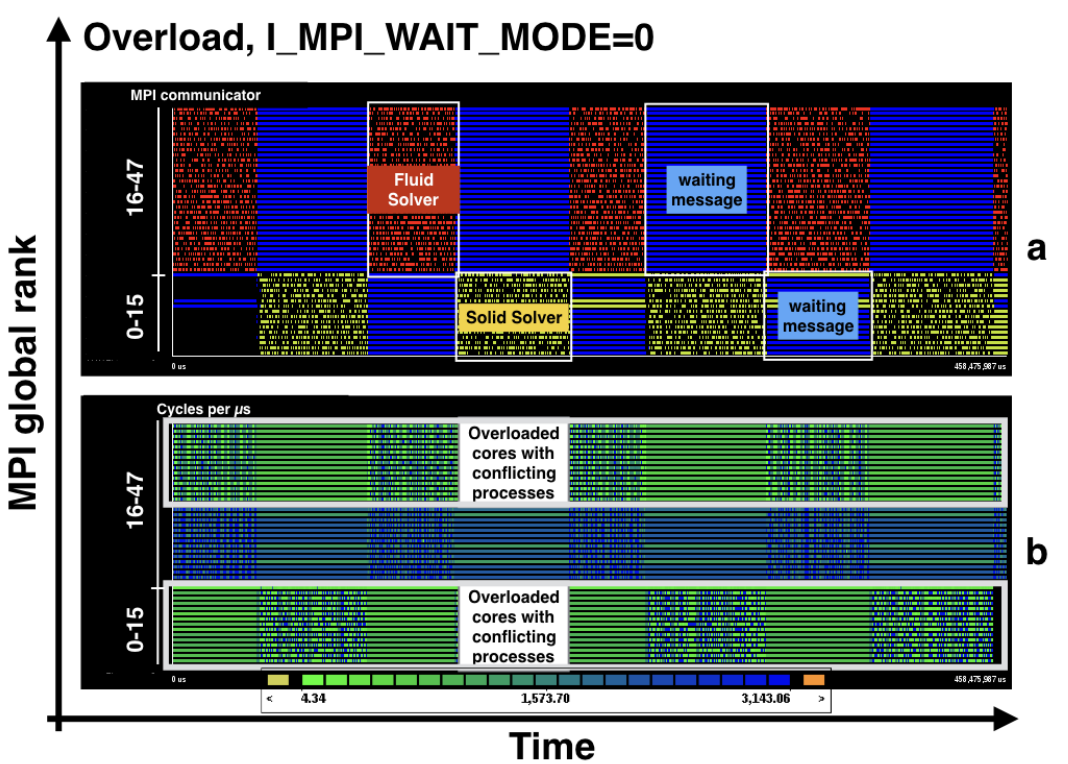

FIG. 17. Trace for an FSI staggered multicode coupled simulation in 32 overloaded cores. 32 MPI processes are used for the fluid code and 16 MPI processes for the solid code. MPI communicator in use (a) and cycles per microsecond used by each MPI process (b). In this case, the waiting code hinders the calculating one by constantly sampling the status of MPI messages. A dramatic increase in the overall execution time with respect to the standard nonoverloaded execution is obtained.

as many cycles as when doing useful computations (see Figure 16). The result is a competition between the waiting and the calculating MPI processes, and a dramatic increase of the overall execution time is obtained.

With all the previous in mind, a second idea to improve the resource usage in the multicode coupled simulation is to avoid the constant sampling of the status of the message when waiting for MPI messages. This can be done by activating the MPI wait mode (I_MPI_WAIT_MODE $=1$ ) and means that the MPI processes will not consume as many cycles of their hosting core as when doing useful computations while waiting for messages. The drawback of this wait mode is that the end of the MPI communications can be delayed as the status of the message is checked less frequently. In Figure 18(b), it can be observed that the cycles per microsecond decrease when the codes are waiting for MPI communications. However, although the computing resources are being used as expected, the overall time of execution still increases. In this case, the problem arises from the single code parallelization. Given that the MPI processes aren't constantly sampling the status of the messages, the individual parallel solvers of the fluid and the solid codes communicate more "slowly," which can also be observed from Figure 18(b) in the form of more green regions. In the present example, the fluid code accelerates with respect to the standard execution thanks to the extra computing resources available, but the solid code decelerates due to the slowdown of the communication process. The overall result is that the coupled simulation time increases; see Figure 20 for easy comparison.

The ideal situation would be that each MPI process turns on the MPI wait mode when receiving MPI messages coming from the other code and turns it off when 


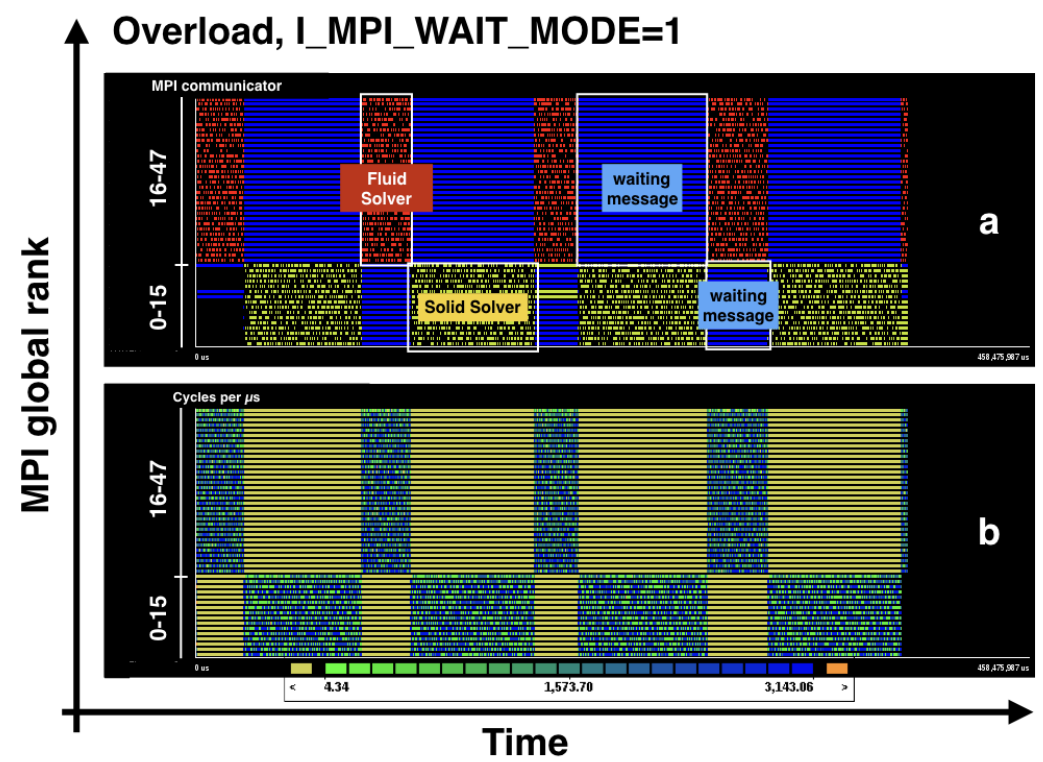

FIG. 18. Trace for an FSI staggered multicode coupled simulation in 32 overloaded cores. 32 MPI processes are used for the fluid code and 16 MPI processes for the solid code. MPI communicator in use (a) and cycles per microsecond used by the MPI process (b). In this case, the MPI processes aren't constantly sampling the status of the messages, so the individual parallel solvers of the fluid and the solid communicate "slowly." The overall result is that the coupled simulation time increases compared to the standard nonoverloaded execution.

waiting to receive MPI messages from its own code. This is not a straightforward task, but it can be done using the dynamic load balance (DLB) library developed at the Barcelona Supercomputing Center (BSC) [19], which avoids the constant sampling of the status of the message only for the intercode communications though a special "DLB barrier." The results are shown in Figure 19; the desired behavior is at last achieved, and a very significant improvement in the use of the resources and the parallel execution time of the coupled simulation is observed. Both codes profit from the extra available resources, and the communications of the individual solvers remain efficient. A general comparison between the strategies tried is shown in Figure 20; note that the time scale is the same for all the execution strategies and a gain of $34 \%$ was achieved in case (d) with respect to the standard case (a).

Finally, a strong scaling test was carried out using the overloaded configuration with the DLB barrier activated in order to test the parallel performance of the multicode approach. The meshes employed have $19 M$ elements for the fluid problem and $9.8 \mathrm{M}$ elements for the solid problem. In the test, there were three time steps with four coupling iterations; each one was simulated. In order to obtain the most reliable measurement of the communications and computations time ratio, two algorithmically equivalent (sequential and parallel execution) solvers were employed: the conjugated gradient (CG) solver for the continuity equation and the Euler equation and the GMRES solver for the momentum equation of the fluid flow problem. As a starting point, six computing nodes (96 cores) were used and increased up to 48 computing nodes (768 cores). The configurations for the overloaded coupled problem used are listed in Table 2. 


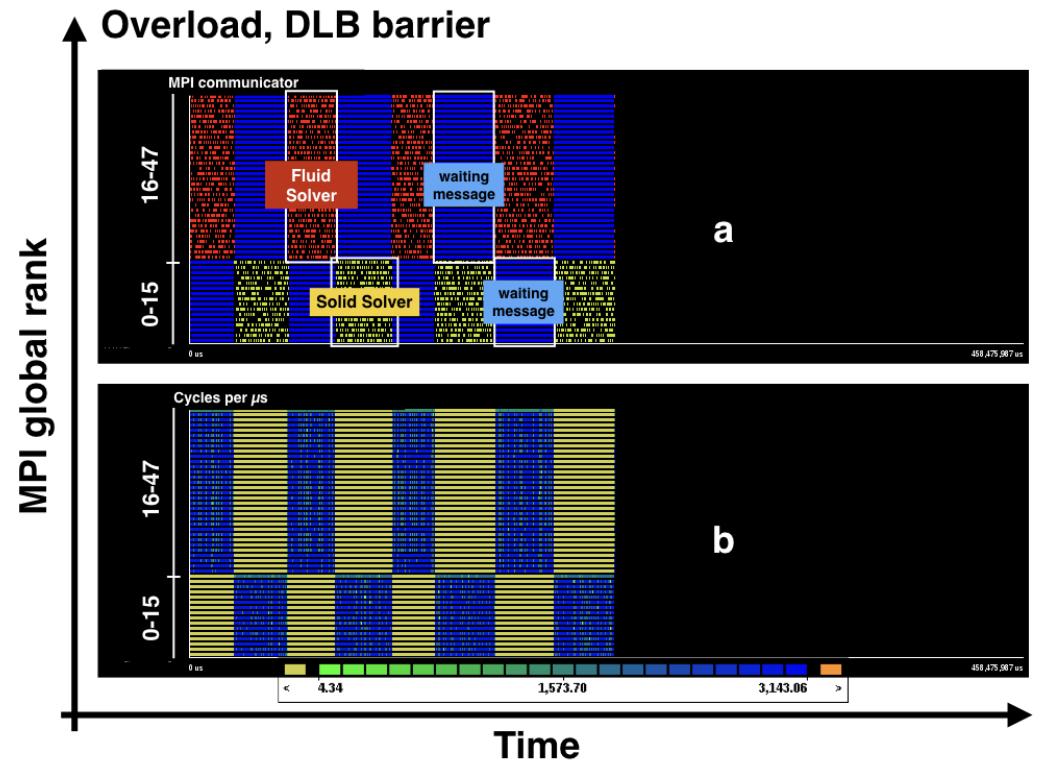

FIG. 19. Trace for an FSI staggered multicode coupled simulation in 32 overloaded cores with 32 MPI parallel processes for the fluid code and 16 MPI processes for the solid code using the DLB library. MPI communicator in use (a) and cycles per microsecond used by the MPI process (b). In this case, the MPI waiting processes aren't constantly sampling the status of the messages from the other code. A very significant improvement in the use of the resources and the execution time of the coupled simulation is observed. Both codes profit from the extra available resources, and the communications of the individual solvers remain efficient.

Results are displayed in Figure 21 and show a satisfactory parallel efficiency, ranging from $98 \%$ with 12 nodes and down to $68 \%$ with 48 . Note, however, that the load per MPI process on the last point is only of 25,000 elements in the fluid solver and 20,000 in the solid one, which is below the normal optimal load used with our code. The coupling setup time was found to increase with the number of nodes used and ranged from $2 s$ to $7 s$.

6. Conclusions. A parallel and efficient multicode coupling framework for FSIHPC problems has been presented. The coupled problem is casted in algebraic matrix form, which is interesting because it makes the framework easy to generalize to other multiphysics problems. The relevant implementation aspects to get a point-to-point communication scheme between the codes (which is important to minimize the effect of the coupling on the parallel performance) are covered, and two well-established coupling algorithms and acceleration schemes are used. The resultant FSI solver is tested in three different examples containing relevant aspects of FSI problems in different application areas. It has good agreement with previously published numerical and experimental results, demonstrating the robustness of the presented approach. Moreover, the performance of the multicode execution has been studied in detail. A strategy based on overloading the available cores in order to minimize idle times has been presented. A careful treatment of the MPI mode has been required in order to avoid resource competition between the active and idle processes. The optimizations presented have resulted in a $34 \%$ reduction of the time integration for a test based on a real simulation of an FSI interaction for blood flow in the aorta. Finally, we have 


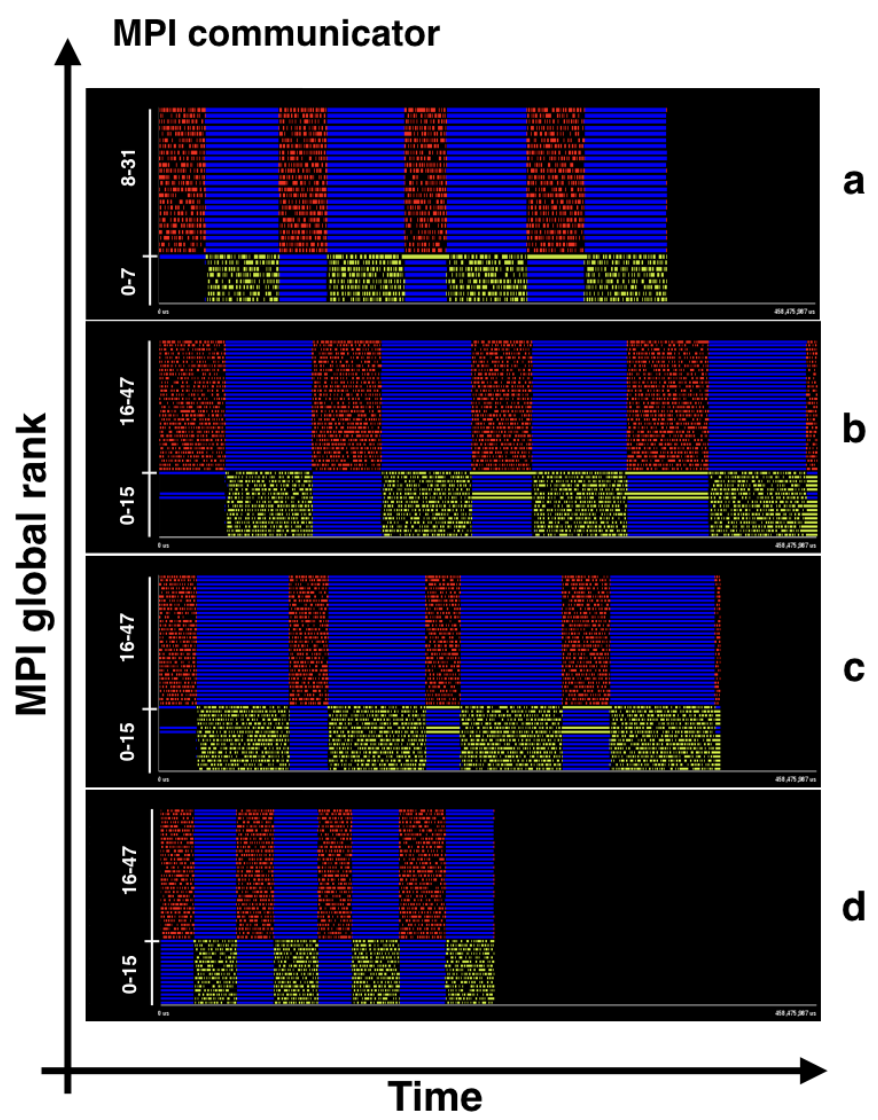

FIG. 20. General comparison of the execution techniques employed for an FSI staggered multicode coupled simulation using 32 computing cores. (a) Standard nonoverloaded simulation; (b) overload without MPI wait mode inactive; (c) overload with MPI wait mode active; (d) overload using the DLB library. The time scale is the same for all the execution strategies.

TABLE 2

Strong scaling resources use for the strong scaling test of the overloaded coupled simulation.

\begin{tabular}{l|c|c|c|c}
\hline Computing nodes (cores) & $6(96)$ & $12(192)$ & $24(384)$ & $48(768)$ \\
\hline MPI processes fluid problem & 96 & 192 & 384 & 768 \\
MPI processes solid problem & 64 & 128 & 256 & 512 \\
Total MPI processes & 160 & 320 & 640 & 1280 \\
\hline
\end{tabular}

attested to a good strong scalability of the multicode execution using up to 1280 MPI processes on 768 cores.

Acknowledgments. This work was achieved using the PRACE Research Infrastructure resources at the Marenostrum (BSC) supercomputer. We thank Dr. Artur Evangelista from the Institut de Recerca Hospital Universitari Vall d'Hebron, Universitat Autònoma de Barcelona, Spain, for access to the medical images and data. 


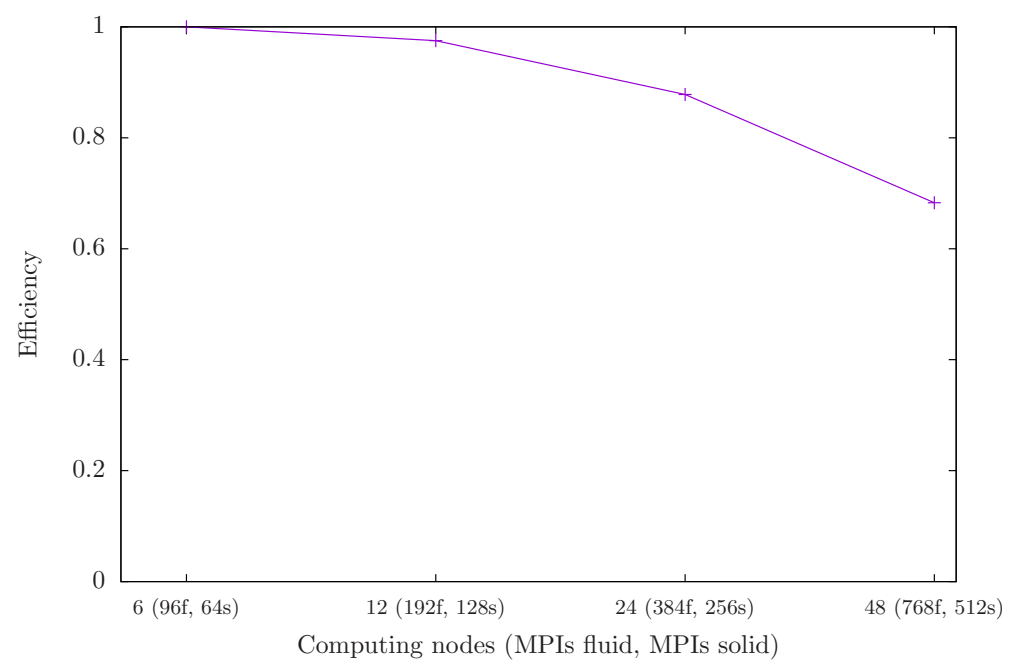

FIG. 21. Strong scaling test results. Efficiencies of 0.98 and 0.88 are obtained for the first and second cases, respectively, and an efficiency of 0.68 for the last case, which was expected given that there are not enough elements for each MPI process (about 25,000 for the fluid problem and 20,000 for the solid problem).

\section{REFERENCES}

[1] M. Avila, A. Folch, G. Houzeaux, B. Eguzkitza, L. Prieto, and D. Cabezón, A parallel CFD model for wind farms, Procedia Comput. Sci., 18 (2013), pp. 2157-2166.

[2] A. T. BARKer AND X.-C. CAI, Scalable parallel methods for monolithic coupling in fluidstructure interaction with application to blood flow modeling, J. Comput. Phys., 229 (2010), pp. 642-659.

[3] Y. Bazilevs, J. Gohean, T. Hughes, R. Moser, and R. Zhang, Patient-specific isogeometric fluid-structure interaction analysis of thoracic aortic blood flow due to implantation of the Jarvik 2000 left ventricular assist device, Comput. Methods Appl. Mech. Engrg., 198 (2009), pp. 3534-3550.

[4] Y. Bazilevs, K. Takizawa, and T. E. Tezduyar, Front matter, in Computational FluidStructure Interaction: Methods and Applications, John Wiley \& Sons, Chichester, UK, 2013, https://doi.org/10.1002/9781118483565.fmatter.

[5] H.-J. Bungartz, F. Lindner, B. Gatzhammer, M. Mehl, K. Scheufele, A. Shukaev, and B. UEKERMANn, preCICE-a fully parallel library for multi-physics surface coupling, Comput. \& Fluids, 141 (2016), pp. 250-258.

[6] H. Calmet, A. M. Gambaruto, A. J. Bates, M. Vázquez, G. Houzeaux, and D. J. Doorly, Large-scale CFD simulations of the transitional and turbulent regime for the large human airways during rapid inhalation, Comput. Biol. Med., 69 (2016), pp. 166-180.

[7] E. Casoni, A. Jérusalem, C. Samaniego, B. Eguzkitza, P. LaFortune, D. Tuahuanto, X. SÁez, G. Houzeaux, and M. VÁzquez, Alya: Computational solid mechanics for supercomputers, Arch. Comput. Methods Eng., 22 (2015), pp. 557-576.

[8] P. Causin, J. Gerbeau, and F. Nobile, Added-mass effect in the design of partitioned algorithms for fluid-structure problems, Comput. Methods Appl. Mech. Engrg., 194 (2005), pp. 4506-4527.

[9] O. Colomés, S. Badia, R. Codina, And J. Principe, Assessment of variational multiscale models for the large eddy simulation of turbulent incompressible flows, Comput. Methods Appl. Mech. Engrg., 285 (2015), pp. 32-63.

[10] W. DetTmer And D. Perić, A computational framework for fluid-structure interaction: Finite element formulation and applications, Comput. Methods Appl. Mech. Engrg., 195 (2006), pp. 5754-5779.

[11] B. Eguzkitza, G. Houzeaux, R. Aubry, H. Owen, and M. Vázquez, A parallel coupling strategy for the chimera and domain decomposition methods in computational mechanics, Comput. \& Fluids, 80 (2013), pp. 128-141.

Copyright $@$ by SIAM. Unauthorized reproduction of this article is prohibited. 
[12] O. Estruch, O. Lehmkuhl, R. Borrell, C. Segarra, and A. Oliva, A parallel radial basis function interpolation method for unstructured dynamic meshes, Comput. \& Fluids, 80 (2013), pp. 44-54, https://doi.org/10.1016/j.compfluid.2012.06.015.

[13] S. Étienne, A. Garon, and D. Pelletier, Perspective on the geometric conservation law and finite element methods for ALE simulations of incompressible flow, J. Comput. Phys., 228 (2009), pp. 2313-2333.

[14] Extrae, Barcelona Supercomputing Center, https://tools.bsc.es/extrae, 2017.

[15] C. FARHAT AND M. LESOINne, Two efficient staggered algorithms for the serial and parallel solution of three-dimensional nonlinear transient aeroelastic problems, Comput. Methods Appl. Mech. Engrg., 182 (2000), pp. 499-515.

[16] C. Farhat, M. Lesoinne, And P. LeTallec, Load and motion transfer algorithms for fluid/structure interaction problems with non-matching discrete interfaces: Momentum and energy conservation, optimal discretization and application to aeroelasticity, Comput. Methods Appl. Mech. Engrg., 157 (1998), pp. 95-114.

[17] M. A. F. Fernández And J.-F. Gerbeau, Algorithms for Fluid-Structure Interaction Problems, Springer Milan, Milano, Italy, 2009, pp. 307-346, https://doi.org/10.1007/ 978-88-470-1152-6_9.

[18] C. Förster, W. A. Wall, And E. Ramm, Artificial added mass instabilities in sequential staggered coupling of nonlinear structures and incompressible viscous flow, Comput. Methods Appl. Mech. Engrg., 196 (2007), pp. 1278-1293.

[19] M. Garcia, J. Labarta, And J. Corbalan, Hints to improve automatic load balancing with LeWI for hybrid applications, J. Parallel Distrib. Comput., 74 (2014), pp. 2781-2794, https://doi.org/10.1016/j.jpdc.2014.05.004.

[20] B. Gatzhammer, Efficient and Flexible Partitioned Simulation of Fluid-Structure Interactions, Ph.D. thesis, Technische Universität München, Institut für Informatik, Garching bei München, Germany, 2014.

[21] M. Gee, U. KÜtTher, AND W. Wall, Truly monolithic algebraic multigrid for fluid-structure interaction, Internat. J. Numer. Methods Engrg., 85 (2011), pp. 987-1016.

[22] S. Gövert, D. Mira, M. Zavala-Ake, J. Kok, M. Vázquez, and G. Houzeaux, Heat loss prediction of a confined premixed jet flame using a conjugate heat transfer approach, Int. J. Heat Mass Transf., 107 (2017), pp. 882-894, https://doi.org/10.1016/j.ijheatmasstransfer. 2016.10.122.

[23] M. HEIL, An efficient solver for the fully coupled solution of large-displacement fluid-structure interaction problems, Comput. Methods Appl. Mech. Engrg., 193 (2004), pp. 1-23.

[24] G. Houzeaux, R. Aubry, and M. Vázquez, Extension of fractional step techniques for incompressible flows: The preconditioned orthomin(1) for the pressure Schur complement, Comput. \& Fluids, 44 (2011), pp. 297-313.

[25] G. Houzeaux, J. C. Cajas, M. Discacciati, B. Eguzkitza, A. Gargallo-Peiró, M. Rivero, AND M. VÁzQUEZ, Domain decomposition methods for domain composition purpose: Chimera, overset, gluing and sliding mesh methods, Arch. Comput. Methods Eng., 24 (2017), pp. 1033-1070.

[26] G. Houzeaux and J. Principe, A variational subgrid scale model for transient incompressible flows, Int. J. Comput. Fluid Dyn., 22 (2008), pp. 135-152.

[27] B. HüBner, E. Walhorn, AND D. Dinkler, A monolithic approach to fluid-structure interaction using space-time finite elements, Comput. Methods Appl. Mech. Engrg., 193 (2004), pp. 2087-2104.

[28] M. Joosten, W. G. Dettmer, And D. Perić, Analysis of the block Gauss-Seidel solution procedure for a strongly coupled model problem with reference to fluid-structure interaction, Internat. J. Numer. Methods Engrg., 78 (2009), pp. 757-778.

[29] U. KÜTtLeR AND W. A. WALL, Fixed-point fluid-structure interaction solvers with dynamic relaxation, Comput. Mech., 43 (2008), pp. 61-72.

[30] M. Lesoinne AND C. FARHAT, Geometric conservation laws for flow problems with moving boundaries and deformable meshes, and their impact on aeroelastic computations, Comput. Methods Appl. Mech. Engrg., 134 (1996), pp. 71-90.

[31] A. G. Malan And O. F. Oxtoby, An accelerated, fully-coupled, parallel $3 D$ hybrid finitevolume fluid-structure-interaction scheme, Comput. Methods Appl. Mech. Engrg., 253 (2013), pp. 426-438.

[32] J. Martorell, R. Pons, L. Dux-Santoy, J. F. Rodríguez-Palomares, J. J. Molins, and A. Evangelista, 4D-MRI coupled to fluid dynamics simulations to improve patient management, Técnicas Endovasculares, XVIII (2015), pp. 25-30.

[33] A. MASUd, Effects of mesh motion on the stability and convergence of ALE based formulations for moving boundary flows, Comput. Mech., 38 (2006), pp. 430-439.

Copyright (c) by SIAM. Unauthorized reproduction of this article is prohibited. 
[34] D. Mira, M. Zavala-Ake, M. Avila, H. Owen, J. C. Cajas, M. Vazquez, and G. Houzeaux, Heat transfer effects on a fully premixed methane impinging flame, Flow Turbul. Combust., 97 (2016), pp. 339-361.

[35] P. Moireau, N. Xiao, M. Astorino, C. Figueroa, D. Chapelle, C. Taylor, and J.-F. Gerbeau, External tissue support and fluid? Structure simulation in blood flows, Biomech. Model. Mechanobiol., 11 (2012), pp. 1-18.

[36] Paraver, Barcelona Supercomputing Center, https://tools.bsc.es/paraver, 2017.

[37] T. Richter, Fluid-Structure Interactions, Lect. Notes Comput. Sci. Eng. 118, Springer, Cham, 2017, https://doi.org/10.1007/978-3-319-63970-3.

[38] T. E. TeZDUYAR, Finite elements in fluids: Stabilized formulations and moving boundaries and interfaces, Comput. \& Fluids, 36 (2007), pp. 191-206.

[39] T. E. Tezduyar, S. Sathe, R. Keedy, and K. Stein, Space-time finite element techniques for computation of fluid-structure interactions, Comput. Methods Appl. Mech. Engrg., 195 (2006), pp. 2002-2027.

[40] S. Turek And J. Hron, Proposal for numerical benchmarking of fluid-structure interaction between an elastic object and laminar incompressible flow, in Fluid-Structure Interaction, Lect. Notes Comput. Sci. Eng. 53, H.-J. Bungartz and M. Schäfer, eds., Springer, Berlin, 2006, pp. 371-385.

[41] B. Uekermann, J. C. Cajas, B. Gatzhammer, M. M. G. Houzeaux, and M. Vázquez, Towards partitioned fluid-structure interaction on massively parallel systems, in Proceedings of WCCM XI/ECCM V/ECFD VI, 2015.

[42] M. Vázquez, R. Arís, J. Aguado-Sierra, G. Houzeaux, A. Santiago, M. López, P. Córdoba, M. Rivero, and J. C. CAJas, Alya Red CCM: HPC-based cardiac computational modelling, in Selected Topics of Computational and Experimental Fluid Mechanics, Springer, Cham, 2015, pp. 189-207.

[43] M. Vázquez, G. Houzeaux, S. Koric, A. Artigues, J. Aguado-Sierra, R. Arís, D. Mira, H. Calmet, F. Cucchietti, H. Owen, A. Taha, E. D. Burness, J. M. Cela, and M. VAlero, Alya: Multiphysics engineering simulation towards exascale, J. Comput. Sci., 14 (2016), pp. 15-27.

[44] M. Vázquez, B. Koobus, A. Dervieux, and C. Farhat, Spatial Discretization Issues for the Energy Conservation in Compressible Flow Problems on Moving Grids, tech. report, Institut national de recherche en informatique et en automatique, Rocquencourt, France, 2003.

[45] W. A. Wall, S. Genkinger, And E. Ramm, A strong coupling partitioned approach for fluidstructure interaction with free surfaces, Comput. \& Fluids, 36 (2007), pp. 169-183.

[46] S. Wang, B. Khoo, G. R. LIU, And G. X. XU, An arbitrary Lagrangian-Eulerian gradient smoothing method (GSM/ALE) for interaction of fluid and a moving rigid body, Comput. \& Fluids, 71 (2013), pp. 327-347.

Copyright $@$ by SIAM. Unauthorized reproduction of this article is prohibited. 